\title{
Anomalous Delayed Loss of Trapped D-D Fusion Products in TFTR
}

\author{
S.J. Zweben, D.S. Darrow, E.D. Fredrickson, and H.E. Mynick \\ Princeton Plasma Physics Laboratory, Princeton, New Jersey, USA
}

\begin{abstract}
A new anomalous delayed loss of D-D fusion products has been measured at the bottom of the TFTR vessel. This loss is delayed by $\approx 0.2 \mathrm{sec}$ with respect to the usual prompt first-orbit loss, and has a correspondingly lower energy, i.e. about half the fusion product birth energy. This loss process dominates the total fusion product loss measured $90^{\circ}$ below the midplane for plasma currents $I \geq 1.8 \mathrm{MA}$ and major radii near $R=2.45 \mathrm{~m}$, e.g. for recent TFTR supershots. This delayed feature can occur without large coherent MHD activity, although it can be strongly modulated by such activity. Several possible causes for this phenomenon are discussed, but no clear explanation for this delayed loss has yet been found.
\end{abstract}




\section{Introduction}

This paper describes measurements of an anomalous loss of partially thermalized trapped D-D fusion products to the bottom (ion $\nabla B$ direction) of TFTR. This feature is the dominant fusion product loss $90^{\circ}$ below the midplane for plasmas with major radii of $R=2.45 \mathrm{~m}$ and plasma currents $I \geq 1.8 \mathrm{MA}$, while for $I \leq 1.4 \mathrm{MA}$ the first-orbit loss is dominant, as observed previously[1]. The relatively low energy of this loss (about half the fusion product birth energy) and its time delay of $\approx 0.2 \mathrm{sec}$ (with respect to the prompt first-orbit loss) imply that some unknown mechanism is causing a relatively slow leakage of fusion product ions from the plasma. Although this leakage is probably not large enough to cause a significant loss of alpha heating in future D-T tokamaks, it could potentially cause a localized alpha particle heat load which could damage the first wall.

Previous measurements of a non-first-orbit loss of trapped D-D fusion products in TFTR were made using a movable detector located $20^{\circ}$ below the outer midplane[2]. At that location the energy of the "anomalous" loss was near the birth energy and not significantly delayed with respect to the first-orbit loss, in contrast with the results for the $90^{\circ}$ detector described below. That loss was approximately consistent with the calculated stochastic toroidal field (TF) ripple loss[3], which causes the banana tips of high energy trapped ion orbits to diffuse vertically, leading to loss near the outer midplane where the trapped orbits first hit the wall. However, these same calculations of stochastic TF ripple loss predict a negligible TF ripple-induced loss $90^{\circ}$ below the midplane[2], and so apparently can not explain the present anomalous delayed loss at $90^{\circ}$.

The delayed loss feature described below is observed in the $90^{\circ}$ detector only for plasmas with major radii near $R=2.45 \mathrm{~m}$, and not for plasmas with $R=2.6 \mathrm{~m}$ such as those used previously to study of diffusion of counter-passing fusion product ions[4]. The smaller plasmas consistently show this delayed loss at plasma current $\mathbb{1} 1.4 \mathrm{MA}$ over the full range of neutral beam power up to $32 \mathrm{MW}$ (in both L-mode and supershot plasmas). There is usually no observable correlation of this delayed loss with MHD activity, although unusually large MHD activity does cause a coherent modulation and change in the strength of this delayed loss feature.

The basic experimental observations as described in Sec. 2 include data taken during the 1990 run period (Secs. 2.1-2.3), data taken during the 1992 run period after a detector relocation (Sec. 2.4), and the effects of strong MHD activity (Sec. 2.5). A preliminary analysis of potentially relevant loss mechanisms is given in Sec. 3 , and a summary and conclusions are in Section 4. 


\section{Measurements of the Anomalous Delayed Loss at $90^{2}$}

The escaping D-D fusion products are detected using the same experimental set-up described previously[1,4]. The detector element is a 1" $\mathrm{x} 1$ " $\mathrm{ZnS}$ scintillator screen behind a pair of apertures which disperse the incident $\mathrm{MeV}$ ions in one direction according to their gyroradius $p$ (depending on their energy) and in the other direction according to their pitch angle $X$ (depending on their magnetic moment). The 2-D images of scintillator light emission are optically coupled to an intensified CCD camera for image capture and later analysis. A detector analysis code determines a $(p, X)$ grid which is used to interpret these images. For this grid the $p$ coordinate is the centroid of predicted scintillator impacts for an ion of gyroradius $p$ (at $X=90^{\circ}$ ), and $X$ is the orbit's pitch angle measured locally with respect to the co-going toroidal field direction at the detector. Normally $75 \%$ of the scintillator light emission from D-D fusion products is from the 3 $\mathrm{MeV}$ protons and 25\% from the $1 \mathrm{MeV}$ tritons[4]. Since these two ions have identical gyroradii (at birth) they are assumed to behave similarly and are treated together as ions of a common gyroradius.

The measurements described in this section were all taken using a detector $90^{\circ}$ below the outer midplane in the ion $\nabla B$ direction. The data of Secs. 2.1-2.3 were taken during the 1990 TFTR run period when the $90^{\circ}$ detector aperture was located $\approx 4 \mathrm{~cm}$ radially outside and about $120^{\circ}$ toroidally from the edge of the nearest "ICRH limiter" (which are poloidal rings intended to shield the ICRH antennae). After the 1990 run a new poloidal ICRH limiter was installed only $45^{\circ}$ toroidally from this same $90^{\circ}$ detector, which forced a relocation of its aperture to only $\approx 1 \mathrm{~cm}$ below the edge of this new limiter[5]. The data described in Sec. 2.4 were taken during the 1992 experimental run period with this latter configuration.

\subsection{Pitch Angle Distributions}

Typical 2-D scintillator light emission patterns observed at the $90^{\circ}$ detector for $I=2.0 \mathrm{MA}$ plasmas are shown in Fig. 1, where in Fig. 1(a) the plasma major radius was $R=2.45 \mathrm{~m}$ and in Fig. 1(b) it was $R=2.6 \mathrm{~m}$. These two discharges were otherwise nearly identical, with $P=23 \pm 1$ MW of neutral beam injection during $\approx 3.0-4.0 \mathrm{sec}$, neutron emission rates of $1.5 \pm 0.1 \times 10^{16}$ neutrons/sec between 3.3-3.4 sec (the integration time for Fig. 1), and $B=4.9 \mathrm{~T}$ on axis. The contours of Fig. 1 show the raw 2-D images at 9 levels from 10\%-90\% of their peak signals. The light emission patterns for the $\mathrm{R}=2.6 \mathrm{~m}$ in Fig. 1(b) are nearly identical to those analyzed previously[4], which were consistent with the first-orbit loss model without any anomalous loss. 
However, the $(\rho, X)$ pattern for the $R=2.45 \mathrm{~m}$ case in Fig. 1(a) was significantly different, with a peak signal located at a significantly higher $X$ and a lower $\rho$ than for the $R=2.6 \mathrm{~m}$ case. The pitch angle distributions for these same two discharges are directly compared in Fig. 2(a), after averaging over gyroradius centroid positions $\rho=2-11 \mathrm{~cm}$ during $3.3-3.4 \mathrm{sec}$. For the $R=2.6 \mathrm{~m}$ case the peak was at $X=63^{\circ}$, which is similar to the previous $R=2.6 \mathrm{~m}$ case, and agreed with the first-orbit model to within $2^{2}[4]$. However, for the $R=2.45 \mathrm{~m}$ case the first-orbit model predicts the peak to be at $X \approx 59^{\circ}$, as illustrated in Fig. 2(b), clearly disagreeing with the measured peak at $X=70^{\circ}$. Note that the first-orbit model curve in Fig. 2 (b) was normalized to the data at $X \approx 55^{\circ}$, suggesting that the measured $X$ distribution at $R=2.45 \mathrm{~m}$ does contain a first-orbit loss component somewhat smaller than the anomalous component which peaks near $X \approx 70^{2}$.

This apparent two-peak structure of the pitch angle distributions for $R=2.45 \mathrm{~m}$ plasmas can be seen more clearly in the scan of plasma current from $\mathrm{I}=0.8-2.5 \mathrm{MA}$ shown in Fig. 3. For plasma currents $I \leq 1.4 \mathrm{MA}$ the pitch distributions agree well with those expected for first-orbit loss[1], while for currents $I \geq 1.6 \mathrm{MA}$ the second peak at $X \approx 70^{\circ}$ begins to appear and eventually dominates the signal at $\geq 1.8 \mathrm{MA}$. Note that only the shapes of the pitch angle distributions are shown here, while the relative magnitudes vs. current are discussed in Sec. 2.3. For a similar current scan at $R=2.6 \mathrm{~m}$ the pitch distributions showed close agreement with the first-orbit model over $\mathrm{I}=0.6-2.0 \mathrm{MA}[4]$, including an expected decrease in the peak pitch angle with increasing current.

For the $I=2.0 \mathrm{MA}$ case the peak pitch angle of $X \approx 70^{\circ}$ with respect to the local toroidal field corresponds to a $74^{\circ}$ pitch angle with respect to the total magnetic field at the detector, or to a normalized magnetic moment of $\mu / \mu_{0} \approx 0.9$. Thus the anomalous loss feature corresponds to a rather deeply trapped ion rather far from the passing-trapped boundary, which is at $\mu / \mu_{0} \approx 0.7$ for fusion product ions lost at this location (see Sec.3.1 for discussion of the orbit trajectories).

This pitch angle data was integrated over the period $\approx 0.5-1.0 \mathrm{sec}$ after the start of NBI, during which time the distributions were approximately constant. However, the pitch distributions were significantly different earlier during the NBI pulse and after the NBI pulse ended, as shown in Fig. 4(a) for a I=1.8 MA discharge (shown earlier in Fig. 3), with 7.5 MW of NBI from 3.0$4.0 \mathrm{sec}$. About $\approx 0.1 \mathrm{sec}$ after the start of NBI the anomalous loss peak near $X \approx 70^{\circ}$ was relatively small compared to the first-orbit peak near $X \approx 60^{\circ}$, while $\approx 0.1 \mathrm{sec}$ after the NBI was switched off (while the neutron source rate was falling) the anomalous loss peak was relatively larger compared 
to the first-orbit peak. The shapes of these distributions became constant $=0.3-0.4 \mathrm{sec}$ after the start of NBI, as indicated by the time dependence of the gyroradius peak location in Fig. 4(b), plotted along with the $\mathrm{I}=2.0 \mathrm{MA}$ case shown in Fig. 2 .

Another characteristic of the high-current pitch angle distributions at $\mathrm{R}=2.45 \mathrm{~m}$ was a tendency for the anomalous loss feature to increase with NBI power (in the steady state), when compared with the first-orbit loss feature. This is illustrated in Fig. 5 for three $I=2.0 \mathrm{MA}$ cases at $P=7.5,12$, and $25 \mathrm{MW}$. These curves are normalized together at $X \approx 55^{\circ}$ (near the first-orbit loss region), showing how the anomalous feature increases with NBI power. This suggests that the anomalous feature depends upon some plasma property which changes with NBI power.

\subsection{Gyroradius Distributions}

The present detector uses the geometrical dispersion of the $\mathrm{MeV}$ ion orbits through the aperture pair to determine the gyroradius $\rho$, which is defined here as the gyroradius which the incident ion would have had at a $90^{\circ}$ pitch angle. As discussed previously[4], there is a considerable instrumental broadening in this detector due to the finite aperture heights, which spreads orbits of a given gyroradius across the $\rho$-dimension of the scintillator plane. Although the measured distributions will be displayed on a grid which represents the centroid locations of the ion impacts of various gyroradii (see Fig. 1), the measurements should actually be compared to the outputs of a detector simulation code which includes the instrumental resolution.

Fig. 6 shows a comparison between the $\rho$ distributions for an $I=2.0 \mathrm{MA}$ discharge (dominated by the anomalous loss feature) and an $\mathrm{I}=0.8 \mathrm{MA}$ discharge (dominated by first-orbit loss), both cases having $R=2.45 \mathrm{~m}$ at the same toroidal field ( $B=4.8 \mathrm{~T}$ at $R=2.45 \mathrm{~m}$ ), again from the current scan in Fig. 3. Note that the peaks of the corresponding pitch angle distributions are both near $x \approx 70^{\circ}$, which makes the inference of their relative gyroradius distribution (averaged over $\left.X \approx 45^{\circ}-85^{\circ}\right)$ less sensitive to possible systematic errors in the $(p, x)$ grid. Also shown in Fig. 6 are calculated particle impact distributions based on assumed incident gyroradii of 4,5 , and $6 \mathrm{~cm}$. The $I=0.8 \mathrm{MA}$ data fits the curve for $\rho=6 \mathrm{~cm}$, roughly consistent with first-orbit loss expected at $\rho=5.5$ $\mathrm{cm}$ (for $3 \mathrm{MeV}$ protons or $1 \mathrm{MeV}$ tritons). However, the $\mathrm{I}=2.0 \mathrm{MA}$ curve peaks at a significantly lower gyroradius near $\rho=4.5 \pm 0.5 \mathrm{~cm}$. This implies that the average energy of the fusion product loss for this case, normalized to the energy for the prompt first-orbit loss $E_{0}$, is roughly: $\mathrm{E} / \mathrm{E}_{\mathrm{O}} \approx[(4.5 \pm 0.5) / 6]^{2} \approx 55 \pm 15 \%$, i.e. corresponding to protons of $\approx 1.5 \mathrm{MeV}$ instead of the $3 \mathrm{MeV}$ 
expected for the protons birth energy. The large instrumental broadening (as shown by the model curves) and various systematic uncertainties[4] do not allow a direct decomposition of the measured distribution at I=2.0 MA into prompt and delayed energy components, but since the prompt loss should be near the birth energy, it is likely that the anomalous loss had an energy somewhat below half the birth energy.

This trend for the anomalous loss feature to have a lower energy than the first-orbit loss can also be seen in Fig. 7, which shows the gyroradius distributions for three times during the same $\mathrm{I}=1.8 \mathrm{MA}$ discharge shown in Fig. 4 . About $\approx 0.1 \mathrm{sec}$ after the start of NBI the gyroradius distribution is close to that for first-orbit loss at $0.8 \mathrm{MA}$ (Fig. 6), peaking near the $\rho=6 \mathrm{~cm}$ centroid, i.e. appreciably below the peak location measured during the steady-state phase $\approx 3.5-4.0$ sec. About $\approx 0.1 \mathrm{sec}$ after $\mathrm{NBI}$ ends the distribution shifts to an even lower gyroradius, peaking just below the $p=4 \mathrm{~cm}$ centroid. The shape of the gyroradius distribution attains its steady-state shape $\approx 0.3 \mathrm{sec}$ after the start of NBI.

Therefore both the time and energy dependencies of the anomalous loss are consistent with a time delay between the ion's birth and its loss. This estimated time delay of $\approx 0.2 \pm 0.1 \mathrm{sec}$ is approximately the same as the time required for classical collisions to slow $3 \mathrm{MeV}$ protons down to $1.5 \mathrm{MeV}$ in these plasmas with $\mathrm{T}_{\mathrm{e}}(0) \approx 7-8 \mathrm{keV}$ and $\mathrm{n}_{\mathrm{e}}(0) \approx 5-6 \times 10^{13} \mathrm{~cm}^{-3}$, implying that these ions are thermalizing classically while diffusing anomalously. This is qualitatively similar to the results from triton burnup measurements in TFTR[6], which implied a classical slowing down but anomalous diffusion of $1 \mathrm{MeV}$ tritons.

\subsection{Magnitude of the Anomalous MeV Ion Loss}

The total $\mathrm{MeV}$ ion loss to the $90^{\circ}$ detector can be estimated from the total light emission within the $(\rho, X)$ grid shown in Fig. 1 , averaged during the steady-state phase of NBI (typically 3.5-4.0 sec), and normalized by the neutron rate during that time. The relative $\mathrm{MeV}$ ion loss signal obtained in this way for a set of $\mathrm{R}=2.45 \mathrm{~m}$ plasmas is plotted vs. plasma current in Fig. 8. These discharges were chosen to have moderate NBI power ( $P=6-20 \mathrm{MW}$, increasing with current) without large coherent MHD activity in the MeV ion signals (see Sec. 2.5). Some of their pitch angle distributions were shown in Fig. 3.

Note that this analysis procedure does not explicitly correct for the dependence of the light emission on the ion energy. However, this correction should be relatively small, since the scintillator light output for D-D fusion products is relatively insensitive to the ion energy in this 
range, e.g. the signai from protons and tritons with half their birth energy (as inferred in Sec 2.2) would be $\approx 0.7$ times the signal for birth-energy ions[4]. Correction for this effect would imply that the anomalous particle flux (ions/sec) would be slightly larger than indicated below.

Fig. 8 shows that the relative $\mathrm{MeV}$ ion loss decreased by a factor of $\approx 5$ with increasing current over the range $I=0.7-2.5 \mathrm{MA}$, while the expected first-orbit loss decreases by about a factor of ten over this same range. When first-orbit loss calculation is normalized to the data at $I=0.7 \mathrm{MA}$ (where the first-orbit loss is expected to dominate), then the total loss at R1.8 MA appears to be not more than a factor of two above that expected from the first-orbit model. However, there are uncertainties of at least $\pm 50 \%$ in the calculated first-orbit loss at $\geq 1.8 \mathrm{MA}$ (with respect to that at $0.7 \mathrm{MA}$ ), due to uncertainty in the neutron source and plasma current profiles[4], which makes it difficult to draw quantitative conclusions concerning the scaling of the anomalous loss vs. current from this figure alone.

Another approach to isolating the anomalous loss component is to decompose the pitch angle distributions of Fig. 3 into a "first-orbit" part which fits the calculated first-orbit loss pitch angle distribution, and a residual part which represents the anomalous loss peaked near $X \approx 70^{\circ}$. Figure 9 shows the results of such an analysis for the $I=1.4-2.5 \mathrm{MA}$ cases in Fig. 3. The anomalous loss component (in the same units as Fig. 8) appears to increase by about a factor of two between I=1.4 and 2.0 MA, while the first-orbit loss component apparently decreases by a factor of 2.5 over the same range. The surprisingly low first-orbit loss component at I=2.5 MA may be due to the effect of the ICRH limiter, which comes close to intercepting birth-energy orbits entering this detector at low pitch angles at $R=2.45 \mathrm{~m}$ ( $\mathrm{see} \mathrm{Sec}$. 3.1).

The approximate dependence of the anomalous loss signal on the neutral beam power in the narrow current range $\mathrm{I}=1.85-2.0 \mathrm{MA}$ is shown in Fig. 10. There the $\mathrm{MeV}$ ion signal at the peak of the anomalous loss feature (including $a \approx 30 \%$ contribution from first-orbit loss) is plotted vs. NBI power over the range $\mathrm{P}=10-31 \mathrm{MW}$ (after normalizing by the neutron rate during the integration time of 3.5-4.0 sec in discharges with NBI from 3-4 sec). The peak loss signal increased by nearly a factor of two over this power range, while the location of the peak remains nearly constant $\left(p=4.2 \pm 0.5 \mathrm{~cm}\right.$ and $\left.X=68^{\circ} \pm 2^{2}\right)$. Note that the first-orbit part of this peak loss signal may increase by up to $\approx 50 \%$ with NBI due to the Shafranov shift and broadened source profiles, but this would only contribute $\approx 10-20 \%$ to this factor-of-two change. The total loss rate (averaged over $p=2-11$ $\mathrm{cm}$ and $X=45^{\circ}-90^{\circ}$ ) increased similarly with NBI power in high current discharges without large MHD[7]. The discharges with "large MHD" are discussed in Sec. 2.5. 


\subsection{Measurements After Relocation of the $90^{\circ}$ Detector}

After the data of Sec. 2.1-2.3 was taken the $90^{\circ}$ detector was moved radially inward $3.3 \mathrm{~cm}$ to avoid the shadowing effect of a new poloidal limiter installed in 1991. The scintillator material was also changed from $\mathrm{ZnS}(\mathrm{Ag})$ (blue) to $\mathrm{ZnS}(\mathrm{Cu})$ (green), and an improved carbon-composite heat shield was added[5]. Most features of the D-D fusion product loss were similar before and after the change; in particular, the $(\rho, X)$ patterns and time dependences for plasmas with $R=2.6 \mathrm{~m}$ (which did not show any anomalous delayed loss) were nearly the same, as were those for $R=2.45$ m plasmas at $\mathrm{I}<1.4 \mathrm{MA}$. The absolute flux of $\mathrm{D}-\mathrm{D}$ fusions products inferred for these cases was also the same in both years (to within a factor of two uncertainty in the cross-calibration).

The qualitative features of the anomalous delayed loss for $R=2.45 \mathrm{~m}$ plasmas with $\mathrm{I} \geq 1.4$ MA in 1992 were also similar to the earlier data, as illustrated by Figs. 11 and 12. The pitch angle distributions shown in Fig. 11 had a shape close to that predicted for first-orbit loss up to $\approx 0.2 \mathrm{sec}$ after NBI, but then evolved over the next $\approx 0.3 \mathrm{sec}$ to a shape with an additional anomalous loss near $X=70^{\circ}$, i.e. similar to the behavior shown in Figs. 2 and 4. Over this same time interval the gyroradius peak decreased from $\approx 4.5 \mathrm{~cm}$ to $\approx 3.6 \mathrm{~cm}$, as shown in Fig. 12, implying average energy of the decrease to $E / E_{o} \approx 0.6$, i.e. again similar to the 1990 results in Fig. 6.

On the other hand, the detailed shapes of the pitch angle distributions at $\mathbb{R} 1.4 \mathrm{MA}$ were different between 1992 and 1990, as illustrated by Fig. 13 for two $R=2.45 \mathrm{~m}, \mathrm{I}=1.8 \mathrm{MA}$ discharges at $P \approx 7.5 \mathrm{MW}$. The two-peak structure which was evident in Figs. 2 and 3 was not so clear in the 1992 data, which instead had a relatively larger feature near the expected first-orbit loss peak at $X \approx 55-60^{\circ}$. In principle, this could be due either to a decrease in the anomalous delayed loss near $X \approx 70^{\circ}$, or to an increase in the anomalous delayed loss near $X \approx 55-60^{\circ}$. The latter is strongly suggested by the results of Fig. 14, which show a decrease in the average loss energy in the region $X \approx 55-60^{\circ}$ in the 1992 data, but not in the 1990 data (which showed delayed loss only near $X \approx 70^{9}$ ). The implication is that after its repositioning the $90^{\circ}$ detector collected more anomalous delayed loss at low pitch angles near $X \approx 55-65^{\circ}$ than it had previously, presumably because these ions had been lost at the ICRH poloidal limiter in the 1990 run. This inference appears to be consistent with orbit trajectory calculations described in Sec. 3.1.

Further evidence for an increase in the delayed loss after the detector relocation was seen in the dependence of the total loss on plasma current shown for the 1992 data in Fig. 15. When the total loss was normalized to the data at the lowest current, as it was for the 1990 data in Fig. 8, the 
resulting loss at $\mathrm{I}=1.8 \mathrm{MA}$ was about a factor-of-two above the expected first-orbit loss, i.e. higher than the corresponding anomaly in the data from 1990 in Fig. 8. Note that the scatter in the $\mathrm{I}=1.8$ MA data in Fig. 15 was mainly due to a systematic increase in the anomalous delayed loss with NBI power, similar to that shown in Fig. 10, and not due to MHD activity (this 1992 data was taken at low NBI powers of $\mathrm{P}=2.5-12 \mathrm{MW}$ ).

The time dependences of the total D-D fusion product loss for typical moderate-power $R=2.45 \mathrm{~m}$ discharges from 1992 are shown in Fig. 16. In these cases the total loss was monitored by a fast photomultiplier (PM) tube, which was not available in 1990, and the total loss signal was normalized to the neutron rate at $3.1 \pm 05 \mathrm{sec}$, i.e. $0.1 \mathrm{sec}$ after NBI. For the $\mathrm{I}=0.6$ and $1.0 \mathrm{MA}$ cases there was a close proportionality between the $\mathrm{MeV}$ ion loss signal and the neutron rate, as expected for prompt first-orbit loss. For the higher current cases there was a delayed loss starting at $\approx 0.15 \mathrm{sec}$ after NBI, increasing to about a factor-of-two above the expected prompt loss rate after $\approx 0.4 \mathrm{sec}$, and persisting up to $\approx 0.3 \mathrm{sec}$ after the end of NBI.

Examination of the 1992 data from the fast PM monitor also showed no consistent fluctuations in the range $\approx 1 \mathrm{~Hz}<<<20 \mathrm{kHz}$ during the anomalous delayed loss, except during large MHD activity (generally at high NBI powers). For $R=2.6 \mathrm{~m}$ discharges in the 1992 run there was a close proportionality between the $\mathrm{MeV}$ ion loss and the neutron rate for all plasma currents up to 1.8 MA (up to at least $P \approx 15 \mathrm{MW}$ ), i.e. similar to the results of 1990 .

\subsection{Effects of MHD Activity on the Anomalous Loss Feature}

For nearly all beam-heated plasmas with $D 1.4 \mathrm{MA}$ at $\mathrm{R}=2.45 \mathrm{~m}$ the anomalous delayed loss feature in the $90^{2}$ detector remained approximately invariant after $\approx 0.4 \mathrm{sec}$ past the start of NBI, and persisted very reproducibly over the 1990 and 1992 run periods. The exceptions to this reproducible behavior all seemed to occur during discharges with strong MHD activity, when both the magnitude and the $(\rho, X)$ structure of the anomalous loss feature could vary substantially.

The most common effect of large MHD activity was to increase the loss rate within the region near the peak of the anomalous loss feature. The time dependence of the loss within this region $\left(\rho \approx 3-4 \mathrm{~cm}, X \approx 65-70^{\circ}\right)$ is shown in the first part of Fig. 17 for a pair of discharges taken from Fig. 10, one of which had "large MHD". The peak signal increased by over a factor of ten in the MHD-active discharge ( $\# 51263)$ compared to the comparison case (\#51256), while the neurron rate decreased by only $\approx 30 \%$. The shot with "large MHD" had slow $(\approx 0.1-0.3 \mathrm{kHz}) \mathrm{m}=2, \mathrm{n}=1$ activity after $3.25 \mathrm{sec}$ at an unusually large level of $\widetilde{\mathrm{B}}_{\mathrm{r}} \mathrm{B}_{\mathrm{T}} \approx 5 \times 10^{-4}$ (estimated near the $\mathrm{q}=2$ surface), 
along with high-n kink activity, while the comparison shot had a few sawteeth along with $m=n=1$ fishbone activity, as did most of the other discharges of Fig. 10. The shot with "large MHD" in Fig. 17 had a variety of $(p, x)$ loss patterns during the MHD activity, most often with an unusually large high- $\chi$ feature at an unusually low $\rho$ (e.g. as shown at $3.56 \mathrm{sec}$ ), as if the MHD activity were increasing the delayed loss process, although at times the peak loss moved to near the passingtrapped boundary (e.g. at $3.89 \mathrm{sec}$ ). The shot with sawteeth and fishbone activity had relatively normal $(\rho, X)$ distribution for this current and power. Note that this increased loss associated with MHD activity could persist for over $0.5 \mathrm{sec}$, i.e. longer than a $3 \mathrm{MeV}$ proton slowing-down time.

As shown in the first part of Fig. 17, this increased loss associated with large MHD activity often fluctuated from frame-to-frame as viewed by the relatively slow video camera, especially for slowly fluctuating MHD activity (e.g. with a $1 \mathrm{msec}$ gate every $32 \mathrm{msec}$ for Fig. 17). These fluctuations could be seen more clearly using a PM tube, as illustrated for a different pair of discharges in Fig. 18 (both $I=1.75 \mathrm{MA}, \mathrm{R}=2.45 \mathrm{~m}$ and $\mathrm{P} \approx 32 \mathrm{MW}$ ). The PM signals of Fig. 18 again came from the peak loss region in the $(\rho, X)$ image plane, and showed a fluctuating $\mathrm{MeV}$ ion loss in phase wis magnetic fluctuations in the case with large, slow MHD (\#55797), but not in the case with relatively less MHD activity, which had only the usual anomalous loss feature (\#55796).

An apparently different effect of MHD activity on the anomalous loss feature is illustrated in Fig. 19, which compares two discharges at $I=2.0 \mathrm{MA}, P=25 \mathrm{MW}$, and $\mathrm{R}=2.45 \mathrm{~m}$ (similar to that of Fig. 1). In one of these (\#55018) there was apparently a "locked mode" during NBI, apparently caused by an unusual sequence of pellet injection prior to NBI. In this case the anomalous loss feature near $X \approx 70^{\circ}$ apparently disappeared in the $90^{\circ}$ detector during the whole period $\approx 3.5-3.9 \mathrm{sec}$, while the gyroradius distribution during this same period was nearly consistent with first-orbit loss alone $(\rho \approx 5-6 \mathrm{~cm})$. Earlier in this same discharge there was also a period of increased loss near $X \approx 70^{2}$ and $\rho \approx 3-4 \mathrm{~cm}$, similar to that shown in Fig. 17. In fact, during many discharges with large slow MHD activity (when the video camera gating time was chorter than the MHD period) the anomalous loss feature corld also sometimes decrease with respect to its usual (anomalous) level without MHD activity, as shown in Fig. 17 at $3.76 \mathrm{sec}$.

During the 1992 run the effects of large MHD were generally similar to those observed during the 1990 run as described above; however, one interesting result from the 1992 data is shown in Fig. 20. During one of these two $R=2.52 \mathrm{~m} \mathrm{NBI}$ discharges (\#68522) there was a dramatic increase in the anomalous delayed loss feature $\approx 50 \mathrm{msec}$ before a major disruption, when compared with the usually small delayed loss feature in $R=2.52$ discharges (\#69111). This 
increased loss, presumably due to pre-disruptive MHD activity, occurred along with an increased loss sharply localized near the passing-trapped boundary. This shows that the delayed loss feature which is normally small for these $R=2.52 \mathrm{~m}$ plasmas at $90^{\circ}$ can increase there during periods of intense MHD activity, and that MHD activity can cause both a delayed trapped particle loss and also a loss of passing particles across the passing-trapped boundary (which was probably also seen during non-disruptive MHD activity such as in Fig. 17 at $3.89 \mathrm{sec}$ ).

These examples show that the anomalous delayed loss feature seen in the $90^{\circ}$ detector can be strongly modulated by MHD activity. They suggest (but do not prove) that the delayed loss normally observed without any obvious MHD activity (such as described in Secs 2.1-2.4) might be caused by some type of normally unnoticed but very reproducible internal MHD activity (or other perturbation), e.g. very slow $(<1 \mathrm{~Hz}$ ) or locked modes, or possibly very fast modes ( $\mathrm{f}>50$ $\mathrm{kHz}$ ). Additional data on MHD-induced fusion product losses are described elsewhere[7], and theoretical mechanisms for an MHD effect are discussed in Sec. 3.4.1.

\section{Discussion}

This section discusses briefly several topics potentially relevant to an understanding of this anomalous delayed loss feature. However, these discussions should be considered preliminary, since no clear explanation for the experimental observations of Sec. 2 has yet been found.

\subsection{Trajectories of Anomalous Loss Orbits}

The measurement the lost ion's $(\rho, X)$ at the detector allow a fairly precise calculation of the trajectory of the ion's last orbit in the plasma. Although these orbits are useful to help determine the loss mechanism, they can not be used to determine the ion's previous history in the plasma.

The trajectories of three relevant loss orbits for the $90^{\circ}$ detector are shown in Fig. 21(a), each of which has been calculated for the same plasma with $R=2.45 \mathrm{~m}$ and $\mathrm{I}=2.0 \mathrm{MA}$ (similar to that of Fig. 2). One of these orbits is the "fattest hanana" for first-orbit loss of $3 \mathrm{MeV}$ protons, which has a pitch angle of $X=55^{\circ}$ (see Fig. 3). This orbit trajectory misses the nearest ICRH limiter (as it was configured in 1990), and approaches within about a/2 of the center of the outermost flux surface. The other two orbits are at $X=70^{\circ}$, one with an assumed energy of $1 \mathrm{MeV}$ and the other with $3 \mathrm{MeV}$ (which bracket the range of the anomalous loss energy). These two $X=70^{\circ}$ orbits (and all those with intermediate energies) intersect the top of the vessel before the 
completion of one bounce; thus at first sight it is difficult to understand how any previouslyconfined orbits can be lost to the $90^{\circ}$ detector at this pitch angle in these plasmas.

One possible mechanism for this is illustrated in Fig. 21(b), which shows a typical anomalous loss orbit entering the detector at $X=70^{\circ}$ and $E=1.5 \mathrm{MeV}$, along with a confined orbit of the same energy which was started with an upward vertical displacement of the lower banana tip of $\approx 15 \mathrm{~cm}$ with respect to the other $1.5 \mathrm{MeV}$ loss orbit. The outer leg of this confined orbit just barely misses the projection of the ICRH limiter (centered at $R=2.61 \mathrm{~m}$ with a minor radius of 0.99 $\mathrm{m}$ ) near the outer midplane. Thus confined orbits with banana tips just below this one would intersect the wall just below the outer midplane and not at the $90^{\circ}$ detector location.

Therefore the anomalous loss orbits detected at $90^{\circ}$ could be brought there by a relatively large vertical step on the last bounce of a previously confined (and partially thermalized) trapped orbit, while smaller vertical steps whe uld cause the loss to occur nearer the outer midplane, as in stochastic ripple diffusion[2]. Such a is. " $\xi$ vertical step could occur while still conserving the ion's magnetic moment (banana tip major radius) and energy; some possible mechanisms for such a large last step size are discussed in Secs. 3.4.

Note that the real limiter and wall structure in TrTR was actually rather complicated, with 2 poloidal ring limiters at a minor radius of $99 \mathrm{~cm}$ in the 1990 run (and more at the same radius in the 1992 run), various structures at $\approx 103 \mathrm{~cm}$, and a solid wall at $\approx 116 \mathrm{~cm}$. The above estimate of a step size of $\approx 15 \mathrm{~cm}$ is based on numerical orbit calculations which include the 2 RF limiters at their appropriate toroidal angle for the 1990 run. However, given the potential inaccuracies in the orbit calculations (e.g. due to current profile assumptions), and in the alignments of ICRH limiters (about $\pm 0.3 \mathrm{~cm}$ ), it is possible that $\mathrm{a} \approx 10 \mathrm{~cm}$ vertical step size would also be large enough.

The different pitch angle distributions in 1990 and 1992 (see Fig. 13) are probably caused by the differing ICRH limiter and probe geometries. In the configuration of 1990 (with the detector aperture $\approx 4 \mathrm{~cm}$ below the nearest ICRH limiter $120^{\circ}$ away toroidally) the orbits of $3 \mathrm{MeV}$ protons at I=2 MA calculated backward in time from the $90^{\circ}$ detector miss the nearest limiter for all pitch angles $x>30^{\circ}$, whereas for the $1.5 \mathrm{MeV}$ protons all the trajectories with $x<55^{\circ}$ hit the ICRH limiter before approaching the detector. Thus, given the uncertainty of about $\pm 0.3 \mathrm{~cm}$ in the relative positions of the detector and limiters, it is plausible that in 1990 the delayed loss orbits with low pitch angles $X \approx 55-65^{\circ}$ were shadowed by the nearest ICRH limiter. However, in the configuration of the $1992 \mathrm{run}$ (with the detector aperture $\approx 1 \mathrm{~cm}$ below the nearest ICRH limiter $45^{\circ}$ away toroidally), the orbits for all $1.5 \mathrm{MeV}$ (and $3 \mathrm{MeV}$ ) protons at I=1.8 MA missed the nearest 
ICRH limiter for all $X>45^{\circ}$; thus the low- $X$ orbits were more likely to reach the $90^{\circ}$ detector in the 1992 configuration.

In these cases the limiter shadowing effect increased for ions which stayed closer to the magnetic flux surface[5], e.g. for lower energy fusion products, but it also increased with increasing plasma current (i.e. decreasing banana width). For example, in the 1990 configuration the $3 \mathrm{MeV}$ loss orjits at I=2.5 MA with $X \leq 45^{\circ}$ hit the ICRH limiter, along with $1.5 \mathrm{MeV}$ orbits with $X \leq 59^{\circ}$, which may explain the unusually low first-orbit loss at this current shown in Figs. 3 and 9. Note that at the lower currents (IS1.4 MA) all the relevant orbits easily miss the nearest ICRH limiter in both configurations, due to the large deviations of the $\mathrm{MeV}$ ion orbits from the flux surfaces.

The general implication of these trajectory calculations is that poloidal, toroidal, and minor radial locarion of the anomalous $\mathrm{MeV}$ loss can depend sensitively on the relative detector and limiter positioning. This is analogous to the localization of plasma energy loss on limiter leading edges, although the large orbits of these ions can allow them to move much farther into the limiter scrape-off region than the plasma itself.

\subsection{Dependence on the Plasma Major Radius}

The anomalous delayed loss measured $90^{\circ}$ below the outer midplane occurred for plasmas with major radii $R=2.45 \mathrm{~m}$ and not for plasmas with $R=2.6 \mathrm{~m}$. For plasmas within the range 2.45 $\mathrm{m}<\mathrm{R}<2.6 \mathrm{~m}$ the anomalous feature decreased gradually toward larger $\mathrm{R}$ (with respect to the first-orbit loss feature), and in plasmas which were moved in $\mathrm{R}$ during a single discharge the anomalous loss feature varied vs. $R$ in the same way as for the constant- $R$ discharges. For plasmas with smaller $2.3 \mathrm{~m}<\mathrm{R}<2.45 \mathrm{~m}$ there is limited data, but the anomalous loss feature appeared to dominate at a relatively smaller plasma current than for $R=2.45 \mathrm{~m}$ plasmas (e.g. at $\mathrm{I}=1.4 \mathrm{MA}$ instead of $\mathrm{I}=1.8 \mathrm{MA})$.

This sensitivity of the anomalous loss feature to the plasma major radius is probably due to the varying relationship between the escaping fast ions and the vessel wall, rather than to an intrinsically differing loss vs major or minor radius. Plasmas with $R=2.6$ are very nearly concentric with the ICRH limiter structure, so that there is very little space near the outer midplane for the outer legs of trapped ion orbits to circulate while they are moving radially (see Fig. 21). For example, in a $R=2.6 \mathrm{~m}$ plasma with $\mathrm{I}=2.0 \mathrm{MA}$ (in the 1990 configuration) the vertical step size needed to cause a marginally confined $1.5 \mathrm{MeV}$ trapped proton orbit like that in Fig. 21(b) to hit 
the bottom detector at $X \approx 70^{2}$ is $\approx 25 \mathrm{~cm}$, which is larger than the $\approx 15 \mathrm{~cm}$ needed for the $R=2.45 \mathrm{~m}$ plasma. Thus it is less likely that ano ralous delayed loss would be detected at $90^{\circ}$ in $R \approx 2.6 \mathrm{~m}$ plasmas, assuming the same vertical step sizes for both plasmas.

In general, the poloidal intersection angle $\theta$ (below the outer inidplane) of a trapped orbit which was just barely confined within the outer limiter on its last bounce is given by:

$$
\rho_{b}\left[1-\left\{1-\left(\theta / \theta_{\text {tip }}\right)^{2}\right\}^{1 / 2}\right]-\delta r \approx \Delta R[1-\cos \theta]
$$

where $\theta_{\text {tip }}$ is the poloidal angle of the previously confined banana tip, $\rho_{b}$ is the banana width, $\delta r$ is the vertical step size of the banana tip on its last bounce, and $\Delta R$ is the distance between the center of the plasma and the center oi the (circular) wall or limiter (assuming $\Delta R<<R$ ). In the limit of small $\delta r$ for $\theta_{t i p} \approx 1$ the solution is: $\left(\theta_{1} \theta_{\text {tip }}\right)^{2} \approx 2 \delta r /\left(\rho_{b}-\Delta R\right)$, which shows how the intersection angle increases as $\Delta R$ increases. Thus for plasmas wibh larger $R$ the loss should occur at smaller poloidal angles for the same vertical step size $\delta r$.

\subsection{MeV Ion Loss at Other Poloidal Angles}

Measurements were also made of $\mathrm{MeV}$ ion loss using similar detectors at poloidal angles $60^{\circ}$ and $45^{\circ}$, although the analysis is not yet as complete as for the $90^{\circ}$ detector measurements discussed in Sec. 2. For the 1990 run the apertures of the $60^{\circ}$ and $45^{\circ}$ detectors were farther outward radially than that for the $90^{\circ}$ detector, i.e. $\approx 6 \mathrm{~cm}$ and $\approx 8 \mathrm{~cm}$ behind the ICRH limiter radius, respectively (compared to $\approx 4 \mathrm{~cm}$ for the $90^{\circ}$ detector). For the 1992 run the apertures of the $60^{\circ}$ and $45^{\circ}$ detectors were both $\approx 1 \mathrm{~cm}$ behind the shadow of the ICRH limiters (similar to the $90^{2}$ detector).

For the same $\mathrm{R}=2.45 \mathrm{~m}$ discharges which showed an anomalous delayed loss feature at $90^{\circ}$ (i.e. with $R=2.45 \mathrm{~m}$ and $\mathrm{P} \geq 1.4 \mathrm{MA}$ ) the detectors at $60^{\circ}$ and $45^{\circ}$ did not usually show any delayed loss or distinct anomalous loss feature at high $X$ and low $\rho$, but generally had a single loss peak at $a(X, \rho)$ location approximately consistent with first-orbit loss. However, for some plasmas with $\mathrm{R}=2.6 \mathrm{~m}$ and $\mathrm{I} \geq 1.4 \mathrm{MA}$ the detector at $60^{\circ}$ showed a clear loss feature at a high pitch angle (separated from the first-orbit loss at lower pitch angle), while the detector at $45^{\circ}$ had only a single feature approximately consistent with normal first-orbit loss. 
This apparent absence of an anomalous delayed loss feature at poloidal angles of $60^{\circ}$ and $45^{9}$ is sorzeswhat surprising, since it seemed from the trajectory arguments of Sec. 3.1 and 3.2 that the anomatous loss feature would be larger at smaller poloidal angles (since the required vertical step siz: was smaller). However, it is still possible that some anomalous delayed loss was masked by the first-orbit loss feature (especially if this loss was at energies nearer to the first-orbit loss), or that the larger TF ripple at these smaller poloidal angles changed the character of the loss (see Sec. 3.4.2).

During large shaw MHD activity such as shown in Figs. 17 and 19 there were fluctuating MHD-induced increases in the signals at $60^{\circ}$ and $45^{\circ}$ which were often out of phase with the increases seen in the $90^{\circ}$ detector, as if the additional loss caused by the MHD activity was poloidally localized and rotating with the MHD mode. This suggests that the anomalous delayed loss usually seen in the $90^{\circ}$ detector without any large MHD activity might be localized there due to the phasing of some nonnally undetected locked mode in the discharge.

The movable detector at a poloidal angle of $20^{2}$ showed signatures of the expected

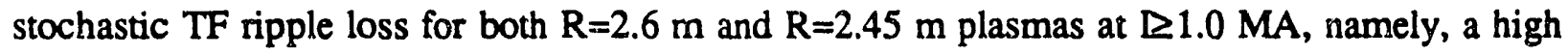
pitch angle feature at nearly the same gyroradius as the first-orbit loss feature[2]. However, this stochastic TF ripple loss signal at $X \approx 60^{\circ}-65^{\circ}$ could also have masked an anomalous loss signal, since the main distinguishing feature would have been a lower gyroradius component, which is not easily resolved in the present detectors. Note that the $20^{\circ}$ detector was normally used at relatively low beam power where the anomalous loss feature was relatively small.

Again, it is important to note that these measurements of anomalous loss can be quite sensitive to radial shadowing by other limiters and even by the probe structure itself. For example, when the $20^{\circ}$ probe was moved radially outward past the ICRF limiter radius, the size its stochastic TF ripple loss feature (with a estimated radial step size per bounce of $\approx 1 \mathrm{~cm}$ ) decreased with respect to the first-orbit loss feature[2]. Therefore comparisons between measurements at different poloidal angles also need to take into account the potentially different effects of limiters and walls on the escaping orbit trajectories. Further analysis of the data and modeling of hypothetical anomalous loss processes will be needed to evaluate the actual poloidal distribution of the anomalous loss.

\subsection{Possible Causes of the Anomalous Delayed Loss}

An anomalous delayed loss of $\mathrm{MeV}$ ions over a timescale of $\tau_{\text {anom }} \approx 0.2 \mathrm{sec}$ corresponds to 
an average anomalous diffusion coefficient of roughly $D_{\text {anom }} \approx \mathrm{a}^{2} / 6 \tau_{\text {anom }} \approx 0.5 \mathrm{~m}^{2} / \mathrm{sec}$, which is much higher than the upper limit previously estimated for the diffusion of counter-passing $\mathrm{MeV}$ ions $\left(D \approx 0.1 \mathrm{~m}^{2} / \mathrm{sec}\right)[4]$, which itself was larger than any collisional neoclassical diffusion rate[4]. This $D_{\text {anom }}$ corresponds to an average radial step size per bounce of only $\delta r \approx 0.1 \mathrm{~cm}$ (for a parallel length $q R=1000 \mathrm{~cm}$ for $1.5 \mathrm{MeV}$ protons), while the trajectory calculations of Sec. 3.1 suggested that the last bounce has a much larger radial step size of $\delta \mathrm{r} \approx 10 \mathrm{~cm}$. Some possible causes for this diffusion are discussed below.

\subsubsection{MHD Effects on Orbit Trajectories}

There are two basic mechanisms by which the magnetic perturbations due to MHD activity can cause radial transport of high energy particle trajectories, namely, the parallel drift of the ion along radially perturbed field lines, and the perpendicular drifts across the field lines. The local radial drift velocity $\delta \mathrm{r} / \mathrm{dt}$ is[8]:

$$
\delta r / \delta t \approx v_{\| 1}\left(\tilde{B}_{\Gamma} / B_{T}\right)+v_{\perp} \text { qn }(\rho / r) \delta
$$

where $\tilde{B}_{r}$ is the local radial magnetic perturbation, $q$ is the magnetic safety factor, $\mathrm{n}$ is the toroidal mode number, $\rho$ is the toroidal gyroradius of the fast ion, and $\tilde{\delta} \approx w / R$ is the ripple amplitude induced by a flux surface displacement " $w$ " (the magnetic island width).

The maximum effect of the first term in Eq. 2 depends upon the maximum parallel length over which the radial perturbation points outward when followed along the fast ion orbit. To accumulate a radial step of $\approx 10 \mathrm{~cm}$ over the last confined bounce period with a parallel length of $\mathrm{qR}$ $\approx 1000 \mathrm{~cm}$ would require a $\tilde{\mathrm{B}}_{\mathrm{T}} / \mathrm{B}_{\mathrm{T}} \approx 10^{-2}$, which appears very unlikely since the normal magnitude of internal magnetic perturbations (without large $M H D$ ) is in the range $B_{r} / B_{T} \approx 10^{-4}[7,8]$. Although a random radial step size implied by the latter level $(\approx 0.1 \mathrm{~cm}$ per bounce) could cause the internal radial diffusion of the confined trapped ions before their last bounce, i.e. $D \approx(0.1$ $\mathrm{cm})^{2} / 10^{-6} \mathrm{sec} \approx 10^{4} \mathrm{~cm}^{2} / \mathrm{sec}$, in order to cause a random step the perturbation must be above a stochastic threshold, which apparently requires $\tilde{\mathrm{B}}_{\mathrm{r}} / \mathrm{B}_{T} \approx 10^{-3}$ for single-mode perturbations[8,9].

The maximum effect of the second term depends upon whether or not the orbit is near the resonant layer of the perturbation. For non-resonant orbits the effective ripple strength $\tilde{\delta}_{n r}$ 
is roughly $\widetilde{\delta}_{n r} \approx\left(\tilde{B}_{\Gamma} / B_{T}\right) /(n-m / q)$, and so over a single bounce time of $\delta t \approx q r / V_{\|}$the magnetic field perturbation needed to cause a step size of $\delta r \approx 10 \mathrm{~cm}$ is again an unrealistically large $\widetilde{B}_{\mathrm{r}} / B_{T} \approx 10^{-2}$, assuming $m=3, n=1, q=4$ and $v_{\|} \approx V_{\perp}$. However, for orbits resonant with the perturbation the effective ripple is related to the half-width of the local magnetic island "w", $\tilde{\delta}_{r} \approx w / R \approx\left(4 q \tilde{B}_{r} / n R q B_{T}\right)^{1 / 2}$. For the parameters above, a $10 \mathrm{~cm}$ step size would require a local $m=3,4 n=1$ perturbation of $\tilde{B}_{r} / B_{T} \approx 2 \times 10^{-3}$, corresponding to $w \approx 12$ and $w / R \approx 5 \times 10^{-2}$, which is higher than the strength of very largest MHD perturbations in TFTR[8]. However, since the step size for resonant perturbations in Eq. 2 scales like $\left(n \widetilde{B}_{\mathrm{r}} / \mathrm{B}_{\mathrm{T}}\right)^{1 / 2}$, the step of $\approx 10 \mathrm{~cm}$ could possibly be explained by an $n=10, \tilde{B}_{r} / B_{T}>2 \times 10^{-4}$ perturbation, as long as the perturbation remains in resonance with the mode (which is unlikely for a smaller-scale mode like this).

Therefore the effects of some hidden MHD activity during seemingly MHD-quiescent plasmas can not easily explain the large last step necessary to bring the escaping orbit to the $90^{\circ}$ detector. However, MHD- iduced effects on confined trapped orbits might be large enough to cause the slow internal dinisic a ate implied by the delayed loss. The observed modulation of the anomalous loss feature during strong MHD activity (Sec. 2.5) is probably due to modulation of the last vertical step, rather than variations of the internal diffusion rate.

\subsubsection{Toroidal Field Ripple Effects}

Toroidal field (TF) ripple can act through at least two different mechanisms to cause radial transport of fast ions. The Goldston-White-Boozer (GWB) stochastic diffusion mechanism causes a collisionless radial step localized near each banana tip of[3]:

$$
\delta r_{G W B} \approx\left(N \pi / \sin \theta_{b}\right)^{1 / 2}(q R / r)^{3 / 2} p \delta \sin \left(N \varphi_{b}\right)
$$

where $\mathrm{N}$ is the number of toroidal field coils ( $\mathrm{N}=20$ in TFTR), $\theta_{b}$ is the poloidal angle of the banana tip $\left(\theta_{b} \approx 90^{\circ}\right.$ here), $\varphi_{b}$ is the toroidal angle of the banana tip, and $\delta$ is the TF ripple near the banana tip. For banana tips at $R \approx 2.45 \mathrm{~m}, z \approx \pm 80 \mathrm{~cm}$, corresponding to the orbits of Fig. $21(\mathrm{~b})$, the vacuum-field TF ripple is $\delta \approx 0.2-0.3 \%[10]$, so the maximum vertical step size for $1.5 \mathrm{MeV}$ protons in a $2 \mathrm{MA}$ plasma at $\mathrm{B}=5 \mathrm{~T}$ is $\delta \mathrm{r}_{\mathrm{GWB}} \approx 2.5-3.5 \mathrm{~cm}$. 
Although this vertical step is apparently too small to explain the $\approx 10 \mathrm{~cm}$ step per bounce needed to cause an ion to be lost at the $90^{\circ}$ detector (see Sec. 3.1), a confined trapped ion can pass through two banana tips before passing near the midplane (see Fig. 21(b)). Therefore, if both the top and bottom banana tips are at the proper toroidal phase angle, the net vertical step per bounce can be up to $\approx 7 \mathrm{~cm}$, which is close to the minimum needed to cause loss at $90^{\circ}$. However, the probability of this correlation is probably small, given the pitch angle averaging of the detectors, such that almost all of the usual GWB loss should be localized within $30^{\circ}$ of the outer midplane, as calculated previously[2].

The other mechanism for radial fast ion transport due to TF ripple involves superbanana trapping inside the ripple wells. This mechanism could readily cause a large vertical step, although it is not clear how the orbit would be de-trapped in order to arrive at the detector with lower pitch angles such as $X=70^{\circ}$. Ripple wells occur when:

$$
\alpha^{*}=(\mathrm{r} / \mathrm{R})|\sin \theta| / \mathrm{Nq} \delta<1
$$

For the banana tip location of Fig. 21(b) used above, $\alpha^{*} \approx 1.5-2$, which appears to be too high for ripple trapping. However, since the ripple strength $\delta$ increases rapidly nearer the edge, ripple wells are expected at $r \approx 90 \mathrm{~cm}$ where $\delta \approx 0.5 \%$, i.e. only about $5 \mathrm{~cm}$ from the lower banana tip of the $90^{\circ}$ detector orbits of Fig. 21(b). Ripple wells occur more readily at smaller poloidal angles, e.g. over the outer $\approx 1 / 3$ of the plasma minor radius at the major radius of the $60^{\circ}$ detector.

Note that since these ripple strengths come from calculations[10] and not from actual TF measurements, it is possible that the effects of coil misalignments, small movements in situ, or stray fields from other coils might cause an increase in the GWB step size or cause the last banana tip to lie within the ripple well at the vessel bottom. However, in the absence of a detailed study of this effect, the tentative conclusion from these estimates is that TF ripple effects probably do not cause the large vertical step on the last bounce discussed in Sec. 3.1, although they could cause trapped orbits to diffuse radially toward that last step. However, it would be surprising if TF ripple effects alone caused the delayed loss, since normally fast ion orbits are either well confined or very rapidly lost due to stochastic TF ripple diffusion[2].

\subsubsection{Pitch Angle Scattering Effects}

Another conceivable mechanism for explaining the anomalous loss to the $90^{2}$ detector is 
classical large-angle scattering, which can change the pitch angle (magnetic moment) of a fast ion while changing its position by only $\approx \rho$. As shown in Fig. $21(\mathrm{c})$, a single $\approx 60^{\circ}$ scattering event can cause the orbit of a confined trapped $1.5 \mathrm{MeV}$ proton to be lost orbit at $X \approx 70^{\circ}$ in the $90^{\circ}$ detector

The Rutherford scattering cross section for large-angle scattering of fast protons on a background hydrogenic species at an angular deflection $\geq \phi$ is[12]: $\sigma=(\pi / 4) b^{2} \cot ^{2}(\phi / 2)$. where $b=e^{2} /\left(M_{0} V^{2} / 2\right)$ and $M_{0}$ is the reduced mass of the colliding ions. Therefore the characteristic rate for a $1.5 \mathrm{MeV}$ proton to be scattered by $\delta \phi \approx 60^{\circ}$ to $90^{\circ}$ on background deuterons at a density of $2 \times 10^{13} \mathrm{~cm}^{-3}$ is $v \approx 10^{-3} \mathrm{sec}^{-1}$. implying that the probability of such a large angle scattering over a characteristic fast proton slowing-down timescale of $\approx 0.2 \mathrm{sec}$ is only $\approx 10^{-4}$. Therefore even though the population of protons born on confined trapped orbits such as that in Fig. 21(c) might be 10 times larger than the population born on a loss orbit such as that also shown in Fig. 21(c), the large angle scattering events would cause only a small enhancement of the firstorbit loss rate on this trajectory. Since the anomalous loss is larger than the expected first-orbit loss on this trajectory, the process of large angle scattering can not explain the anomalous loss. Small angle scattering is more likely; however, small angle scattering would cause a large radial step only near the passing-trapped boundary.

\section{Summary and Conclusions}

An anomalous loss of D-D fusion products was measured using a scintillator detector $90^{\circ}$ below the outer midplane in TFTR. This new loss feature had a significantly lower energy than the usual first-orbit loss ( $\approx 1 / 2$ the birth energy), and was delayed by $\approx 0.2 \mathrm{sec}$ with respect to the usual prompt first-orbit loss. The orbits of the escaping ions lost in this way were fairly deeply trapped, with normalized magnetic moments of up to $\mu / \mu_{0} \approx 0.9$. Some observations and interpretations concerning this anomalous loss process were:

1) The anomalous loss feature increased in strength with respect to the first-orbit loss as the plasma current was increased, e.g. it was comparable to the size of the first-orbit loss at $I \approx 2.0 \mathrm{MA}$, but was negligible compared to the first-orbit loss at currents I $\leq 1.0 \mathrm{MA}$,

2) the anomalous loss feature was visible at the $90^{\circ}$ detector for plasmas with major radii of 
$R=2.45 \mathrm{~m}$ but not for plasmas with $\mathrm{R}=2.6 \mathrm{~m}$, possibly because the escaping ion orbits intersected the wall elsewhere for the plasmas with larger major radii,

3) the observed delay time of $\approx 0.2 \mathrm{sec}$ with respect to the prompt loss was roughly consistent with the time required for $3 \mathrm{MeV}$ protons to lose about half of their energy,

4) the strength of the anomalous loss feature increased by up to $\approx 50 \%$ with increasing NBI power at a fixed current, suggesting that the anomalous loss was influenced by the plasma itself (even without large MHD activity), and,

5) large coherent MHD activity strongly modulated the anomalous loss feature, sometimes causing it to increase but at other times causing it to disappear, suggesting that some low-level MHD activity might cause the delayed loss even in plasmas without large coherent MHD.

A rough analysis of these measurements implied that an average diffusion coefficient of $D_{\text {anom }} \approx 0.5 \mathrm{~m}^{2} / \mathrm{sec}$ was needed to explain this loss of trapped fusion products, which is large compared with the $D<0.1 \mathrm{~m}^{2} / \mathrm{sec}$ previously inferred for passing fusion products[4]. This $D_{\text {anom }}$ is comparable to the thermal plasma heat and particle diffusion coefficients, but for fast fusion products this corresponds to a relatively small step size per bounce of $\delta \mathrm{r} \approx 0.1 \mathrm{~cm}$. However, a much larger vertical step size of $\approx 10 \mathrm{~cm}$ on the ion's last confined bounce was required for a the loss orbit to be detected at $90^{\circ}$ detector in the presence of the poloidal ICRH limiters near the outer midplane. Preliminary evaluations of several possible loss mechanisms concluded that:

1) internal magnetic perturbations with magnitudes $\tilde{\mathrm{B}}_{\mathrm{r}} / \mathrm{B}_{T} \approx 10^{-4}$ are sufficient to cause the average radial step size needed for the delayed loss, although the radial steps for single modes are apparently not stochastic below $\tilde{\mathrm{B}}_{\mathrm{r}} / \mathrm{B}_{\mathrm{T}} \approx 10^{-3}$, and even at that level a single MHD modes can not plausibly explain the required large last step size,

2) the calculated effect of stochastic TF ripple diffusion produced a step size of $\approx 3 \mathrm{~cm}$ at each banana tip in the region of interest, and so would not quite explain the loss at $90^{\circ}$, although the internal radial transport of partially thermalized trapped ions could be caused by this mechanism; and localized TF ripple wells, which could cause large vertical step size, do not quite exist in the region of the last banana tip of the anomalous loss orbit, 
3) large-angle nuclear scattering is not strong enough to cause this anomalous loss process.

Although there is yet no quantitative explanation for the anomalous loss described in this paper, some general conclusions can be drawn from these results:

1) this anomalou; delayed loss will most likely persist for alpha particles in D-T plasmas, since they will be sensitive to similar "single-particle" loss mechanisms and will have a similar thermalization time. However, the magnitude of the global alpha heating loss will probably be $<1-$ $10 \%$ of the confined $\mathrm{MeV}$ ion population, corresponding roughly to the first-orbit loss level in TFTR at I=2 MA[7]. Although such a loss should not affect global alpha heating, it may cause localized heat loads and damage to unprotected wall or divertcr components in the ion $\nabla \mathrm{B}$ direction.

2) the sensitivity of these results to the radial detector relocation implies that the wall impact position of any such "anomalous" MeV alpha loss (including possibie collective alpha effects) can be quite sensitive to relative positions of the plasma, the wall and/or limiters, and the detector itself. This suggests that $\mathrm{MeV}$ ion loss in future DT experiments should ideally be monitored on all exposed limiter surfaces, although practical difficulties such as detector overheating and competing energy loss mechanisms will inevitably limit this information.

3) a large guiding center or bounce-mapping code will be needed to understand the complex MHD and TF ripple loss mechanisms and their mutual interactions in the plasma; such a code should also incorporate the details of the wall and limiter geometry to determine the alpha loss locations.

Future experimental work in this area should clarify the effects of the MHD mode number and its frequency on the observed anomalous loss (particularly for very low frequency modes), and should evaluate its poloidal and toroidal distribution more carefully, as done recently for NBI loss in JT-60U[13]. A search for new theoretical mechanisms for trapped fusion product loss also seems to be warranted by these results. 
Acknowledgements: We thank R. Boivin, G. Hammett, M. Tuszewski, and J.-P. Roubin for helpful discussions, and K. McGuire, J.D. Strachan and K.M. Young for their support of this work, which was performed under US DoE contract \# DE-AC02-76-CHO-3073.

\section{References}

1. Zweben, S.J., Boivin, R.L., Diesso, M., et al, Nucl. Fus. 30 (1990) 1551

2. Boivin, R.L., "Measurements of Charged Fusion Product Diffusion in TFTR" (thesis), PPPL2797 (1991), to be published in Nucl. Fusion (1993)

3. Goldston, R.J., White, R.B., and Boozer, A.H., Phys. Rev. Lett. 47 (1983) 647

4. Zweben, S.J., Boivin, R.L., Chang, C.S., et al, Nucl. Fus. 31 (1991) 2219

5. Zweben, S.J. et al, Rev. Sci. Inst. 63, 564 (1992) and earlier references therein

6. Barnes, C.W., private communication (1992)

7. Zweben, S.J., et al, Proc. 14th International Conference on Plasma Physics and Controlled Nuclear Fusion Research, Wurzburg, Germany, Oct. 1992, paper IAEA-CN-56/A-6-3.

8. Mynick, H.E. and White, R.B., in Theory of Fusion Plasmas, International School of Plasma Physics, Oct. 1988, J. Vaclavik, F. Troyon, E. Sindoni (eds.), pp. 385-400 (1989)

9. Mynick, H.E., "Transport of Energetic Ions by low-n Magnetic Perturbations", Princeton Plasma Physics Laboratory Report \# PPPL-2856, to be published in Phys Fluids (1993)

10. TFTR Magnetics Handbook, EAD-H-1, Princeton Plasma Physics Laboratory

11. Evans, R.D., The Atomic Nucleus, McGraw Hill, New York (1955) pp. 13-16

12. White, R.B. and Mynick, H.E., Phys. Fluids B 1, 980 (1989)

13. Tobita, K., Tani, K., Neyatani, Y, et al, Phys. Rev. Lett. 69, 3060 (199?). 


\section{Figure Captions}

1. Contour plots of the pitch angle vs gyroradius pattern of loss to the $90^{\circ}$ detector for two similar discharges, one at (a) $R=2.45 \mathrm{~m}$ (\#55052) and the other at (b) $R=2.6 \mathrm{~m}$ (\#54315). For both discharges the plasma current was $I=2.0 \mathrm{MA}$, the beam power was $\mathrm{P}=22-23 \mathrm{MW}$, and the data was taken during the time 3.3-3.4 sec. The anomalous loss feature visible occurs at an unexpectedly high pitch angle $X \approx 70^{2}$ and low gyroradius $p \approx 4-5 \mathrm{~cm}$ in (a).

2. Pitch angle distributions for the two $I=2.0 \mathrm{MA}$ cases in Fig. 1 . In (a) is a direct comparison between the shapes of the pitch angle distributions for the $R=2.45 \mathrm{~m}$ and $R=2.6 \mathrm{~m}$ cases, showing the anomalous peak at $X \approx 70^{\circ}$ for the $R=2.45 \mathrm{~m}$ case. In (b) is a comparison between the measured pitch distribution for the $R=2.45 \mathrm{~m}$ case and the calculated first-orbit loss distribution (normalized to the data at $X \approx 55^{\circ}$ ), which shows that the measured peak at $X \approx 70^{\circ}$ is not predicted by the firstorbit loss model.

3. Measured pitch angle distributions and calculated first-orbit loss distributions for $R=2.45 \mathrm{~m}$ plasmas as a function of plasma current. For currents I $\leq 1.4 \mathrm{MA}$ the measured distributions agree fairly well with the first-orbit model, while for currents $\geq 2 \mathrm{MA}$ the anomalous loss feature at $x \approx 70^{2}$ dominates. These measured pitch distributions were taken during the steady-state part of NBI (typically 3.5-4.0 sec in a 3.0-4.0 sec NBI pulse), and the model curves for I $\geq 1.8 \mathrm{MA}$ were normalized to the data at $X \approx 55^{\circ}$. Note that the vertical scales are not comparable from one current to the next (see Fig. 8).

4. Part (a) shows the pitch angle distributions (integrated over $p=2-11 \mathrm{~cm}$ ) just after the start of NBI and just after the end of NBI for the $I=1.8 \mathrm{MA}, \mathrm{R}=2.45 \mathrm{~m}$ discharge shown in Fig. 3(e) (\#54464). Just after the start of NBI the anomalous loss feature at $X \approx 70^{\circ}$ is relatively smaller (compared to the first-orbit loss feature) than it is during the steady-state phase at $\approx 3.5-4.0 \mathrm{sec}$, and just after NBI it is relatively larger. The ratio of the signal at $X \approx 70^{\circ}$ to the signal at $X \approx 60^{2}$ is plotted in part (b) for this case, and also for the $I=2 \mathrm{MA}$ case of Fig. 2, showing that the shapes of these distributions become constant $\approx 0.4 \mathrm{sec}$ after the start of NBI. This behavior implies that the anomalous feature is a delayed loss process.

5. Pitch angle distributions for plasmas with varying NBI power at $R=2.45 \mathrm{~m}$ and $I=2.0 \mathrm{MA}$, taken during the steady-state part of the discharge and nomalized in their first-orbit regions at $X \approx 55^{\circ}$. The discharge with the lowest NBI power of $7.5 \mathrm{MW}$ (\#54468) had the smallest 
anomalous feature, while those with $12 \mathrm{MW}$ and $25 \mathrm{MW}$ (\#54472 and 55050, respectively) had a larger anomalous loss peak, relative to the first-orbit feature at lower pitch angle. The total $\mathrm{MeV}$ ion loss (normalized by the neutron rate) increased by $\approx 40 \%$ over this power range.

6. Gyroradius distributions for $I=0.8 \mathrm{MA}$ and $\mathrm{I}=2.0 \mathrm{MA}$ plasmas (integrated over $X \approx 45-90^{\circ}$ during 3.5-4.0 sec) compared with model calculations for assumed gyroradii of $p=4,5$, and $6 \mathrm{~cm}$. The $I=0.8 \mathrm{MA}$ case fits fairly well the expected $\rho=5.5 \mathrm{~cm}$ for prompt first-orbit loss, while for the $I=2.0 \mathrm{MA}$ case the total loss is best fit by an assumed $p \approx 4-5 \mathrm{~cm}$, implying an average loss energy of $\mathrm{E} / \mathrm{E}_{\mathrm{o}} \approx 0.5-0.6$ compared to the birth energy. The broadening due to instrumental effects causes the calculated distributions to be spread over a range of gyroradius centroid locations.

7. Gyroradius distributions (integrated over $X \approx 45-90^{\circ}$ ) vs. time during the same $I=1.8 \mathrm{MA}$ discharge as for Fig. 4. Just after the start of NBI the gyroradius distribution is similar to that for the first-orbit loss at 0.8 MA shown in Fig. 6, while just after NBI ends the peak of the gyroradius distribution occurs at an even lower $\rho$ than during $=3.5-4.0 \mathrm{sec}$. This confirms the delayed nature of this loss.

8. Total $\mathrm{MeV}$ ion signal vs. plasma current for discharges with $\mathrm{R}=2.45 \mathrm{~m}$ (in the range $\# 53360$ 55362). The ordinate is the total scintillator light output during the period from 3.5-4.0 sec during $\mathrm{NBI}$, normalized by the total neutron rate during that time. The relative loss between $\mathrm{I}=0.7-1.4$ MA follows the first-orbit model curve fairly well (as calculated for discharges in this sequence), but the measured loss between I=1.4 and 2.5 MA decreases less than expected from the first-orbit loss model.

9. Approximate decomposition of the MeV ion pitch angle distributions of Fig. 3 into a "firstorbit" part (with the shape of the calculated first-orbit loss distribution as normalized to the data at $X \approx 55^{\circ}$ ) and ant anomalous part (the remaining signal centered near $X=70^{\circ}$ ). Over the range $I=1.4$ 2.0 MA the anomalous loss signal appears to increase by about a factor of two, while the first-orbit part decreases by a factor of $\boldsymbol{\approx 2 . 5}$. There is also a systematic change in the anomalous loss component with NBI power (not shown here), and a possibility that the data at $I=2.5 \mathrm{MA}$ is affected by shadowing from the ICRH limiter.

10. The effect of NBI power on the relative $\mathrm{MeV}$ ion loss signal measired near the peak of the anomalous loss feature for a set of $I=1.85-2.0 \mathrm{MA}$ discharges (averaged over 3.5-4.0 $\mathrm{sec}$ in discharges with NBI from 3-4 sec). The neutron-normalized peak loss increases slowly with NBI 
power, which is not expected for first-orbit loss, while the pitch distributions change as shown in Fig. 5.

11. Time dependence of the pitch angle distribution measured for a typical $R=2.45 \mathrm{~m}$ plasma during the 1992 run period (averaged over $\rho \approx 2-11 \mathrm{~cm}$ ). The pitch angle distribution is consistent with the expected first-orbit loss distribution up to $\approx 0.2 \mathrm{sec}$ after NBI starts, but after $\approx 0.3-0.4 \mathrm{sec}$ the discributions show an anomalous delayed loss feature near $X \approx 70^{2}$ qualitatively similar to that seen during the 1990 run period.

12. Peak of the gyroradius distribution (in terms of its centroid location) vs. time (averaged over $X=45-90^{\circ}$ ) for a series of $R=2.45 \mathrm{~m}$ discharges during the 1992 run period. The gyroradius distribution remains approximately constant in time for I $\leq 1.0 \mathrm{MA}$ as expected for first-orbit loss, but for $1 \geq 1.4 \mathrm{MA}$ there is a decrease in the peak gyroradius past $\approx 0.2 \mathrm{sec}$ after NBI, which is at least qualitatively similar to the gyroradius distributions seen in the 1990 run (Fig. 6).

13. Comparison of the pitch angle distributions measured by the $90^{\circ}$ detector for 1990 and 1992 discharges, both at at $1.8 \mathrm{MA}, R=2.45 \mathrm{~m}$, and $P=7.5 \mathrm{MW}$ (averaged over $p \approx 2-11 \mathrm{~cm}$ during 3.5$4.0 \mathrm{sec})$. There is a significant difference in the shape of the two distributions, which is attributed to relatively more anomalous delayed loss at low pitch angles $\left(X=55-65^{\circ}\right)$ in the 1992 run, after a repositioning of the $90^{\circ}$ detector aperture.

14. Comparison of the gyroradius distributions measured by the $90^{2}$ detector within the pitch angle region $X=55-65^{\circ}$ for the 1990 and 1992 discharges shown in Fig. 13. There is $c$ significant decrease in the peak of the gyroradius distribution vs. time in this range for the 1992 data, but not for the 1990 data, indicating that there is a significant anomalous delayed loss at these low pitch angles in the 1992 data.

15. Total $\mathrm{MeV}$ ion loss signal vs. plasma current for discharges with $\mathrm{R}=2.45 \mathrm{~m}$ for the 1992 run (in the range \#64429-64462), similar to the plot for the 1990 data in Fig. 8. The relative loss between $\mathrm{I}=0.6-1.8 \mathrm{MeV}$ does not decrease as much as expected from the first-orbit model (as calculated for discharges in this sequence), and appears to decrease less than the corresponding data from 1990.

16. Time dependences of the total loss signal from the $90^{\circ}$ detector for $R=2.45 \mathrm{~m}$ discharges from the 1992 run (measured using a fast photomultiplier detector). For $\mathrm{I}=0.6$ and 1.0 MA the measured loss is proportional to the neutron rate, while for $\mathrm{I}=1.4$ and $1.8 \mathrm{MA}$ there is a significant 
delayed loss component starting at $\approx 0.15 \mathrm{sec}$ after NBI, and lasting up to $\approx 0.3 \mathrm{sec}$ after NBI ends. For each current the $\mathrm{MeV}$ ion loss is normalized to the neutron rate at $3.1 \pm 0.05 \mathrm{sec}$.

17. Time dependences of the $\mathrm{MeV}$ ion loss signals for two different discharges (from the 1990 run) with $R=2.45 \mathrm{~m}, \mathrm{I}=1.85 \mathrm{MA}$, and $\mathrm{P} \approx 22 \mathrm{MW}$. One of these has large $\mathrm{m}=2, \mathrm{n}=1 \mathrm{MHD}$ activity (\#51263), while the other doesn't (\#51256). During the MHD activity the instantaneous MeV ion loss can increase by over a factor of 10 , while the neutron rate is reduced by only $<30 \%$. The pitch angle and gyroradius distributions vary considerably vs. time during the discharge with MHD activity, sometimes showing a large increase in the delayed loss feature at $X \approx 70^{\circ}$ and an unusually low gyroradius distribution peaked at $\rho \approx 3 \mathrm{~cm}$ (e.g. at $3.56 \mathrm{sec})$.

18. Time dependences of the $\mathrm{MeV}$ ion loss signal near the peak of the anomalous delayed loss feature for a discharge with large low frequency MHD activity (\#55797), compared to a discharge with much smaller MHD activity (\#55796). The peak MeV ion loss signal in \#55797 increases by about a factor of two with the onset of the MHD activity at $\approx 3.35 \mathrm{sec}$ and fluctuates in phase with the $\approx 0.5 \mathrm{kHz}$ magnetic loop signals, while it does not appreciably increase or fluctuate with the smaller $\approx 5 \mathrm{kHz}$ fluctuations in \#55796 (ncte that the magnetic signals shown are proportional to $\mathrm{dB} / \mathrm{dt}$ ). This fusion product loss signal comes from a PM tube monitoring the peak emission region with a frequency response of up to $\approx 20 \mathrm{kHz}$.

19. Pitch angle and gyroradius distributions in a discharge without large fluctuating MHD but with a "locked mode" apparently triggered by pre-NBI pellet injection (\#55018), compared to a similar discharge (\#55017) with the usual anomalous delayed loss feature (without pellets). During the =3.5-3.9 sec of $\# 55018$ shown here the high- $\chi$, low- $\rho$ anomalous loss feature is essentially absent, leaving only the first-orbit loss feature. At earlier times in the same discharge $(\approx 3.1-3.3$ sec) the anomalous loss feature is considerably larger than that for the discharge without large MHD (similarly to Fig. 17).

20. Pitch angle vs. gyroradius distributions for an $R=2.52 \mathrm{~m}, \mathrm{I}=2 \mathrm{MA}$ (1992) discharge just before a major disruption (a), compared to a similar discharge without a disruption in (b). About $50 \mathrm{msec}$ prior to disruption the pattern in (a) shows a large delayed loss feature at a relatively high pitch angle and low gyroradius, presumably due to pre-disruptive MHD activity, along with a sharp anomalous loss feature near the passing/trapped boundary at lower pitch and higher gyroradius. The non-disruptive discharge in (b) shows comparatively less delayed loss, as is typical of the large- $R$ plasmas. 
21. Trajectories of possible anomalous loss orbits of $\mathrm{MeV}$ protons to the $90^{\circ}$ detector for an $\mathrm{I}=\mathbf{2 . 0}$ MA, $R=2.45 \mathrm{~m}$ discharge (\#54472). In (a) are superimposed the trajectories of a "fattest banana" first-orbit loss proton ( $X=55^{\circ}$ for a $3 \mathrm{MeV}$ proton), and the loss trajectories for $1 \mathrm{MeV}$ and $3 \mathrm{MeV}$ protons at $X=70^{\circ}$. In (b) is an anomalous loss trajectory for a $1.5 \mathrm{MeV}$ proton at $X=70^{\circ}$ (near the peak of the anomalous loss region), superimposed on a confined trapped orbit of a $1.5 \mathrm{MeV}$ proton with its banana tip at the same $R$ which just misses the outer $R F$ limiter. In (c) is a confined trapped $1.5 \mathrm{MeV}$ proton orbit with a midplane pitch angle $60^{\circ}$ different from the anomalous loss orbit, which could occasionally scatter into the anomalous loss orbit at $X=70^{2}$. 


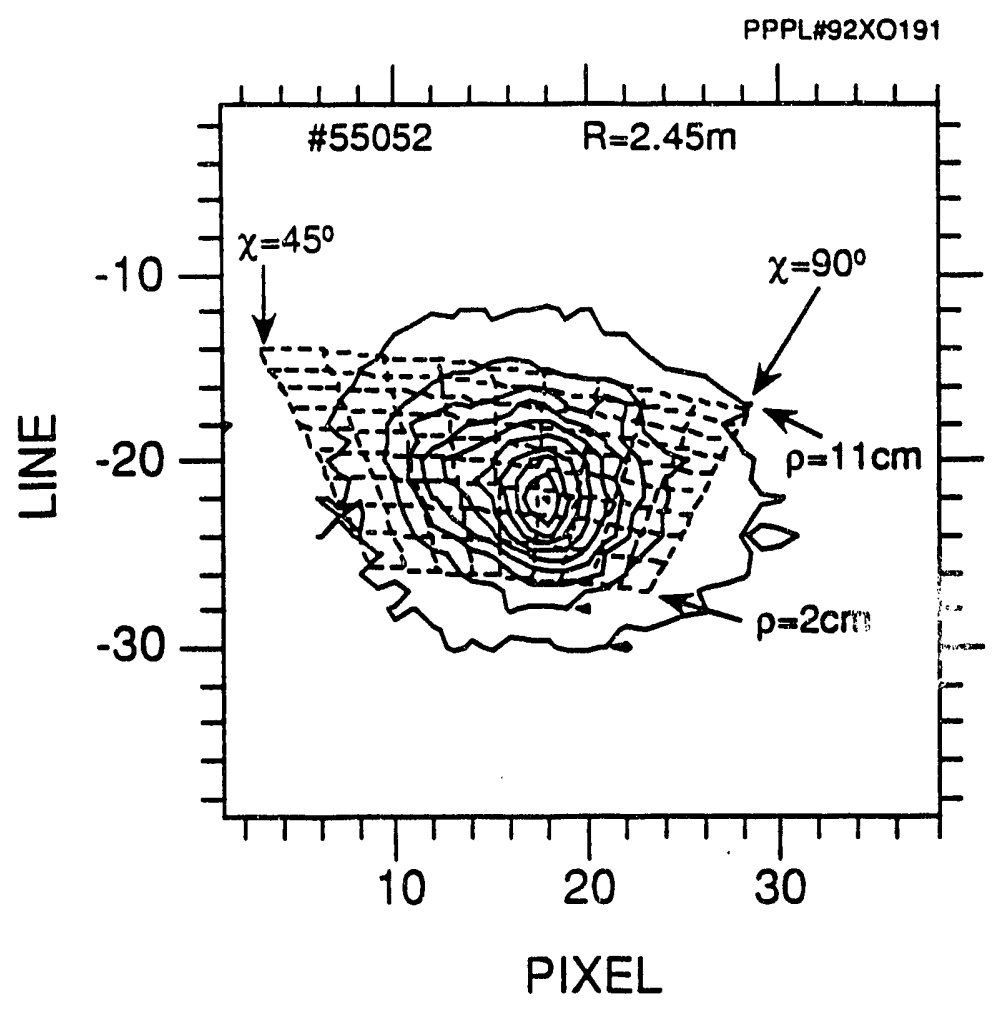

Fig. 1(a)

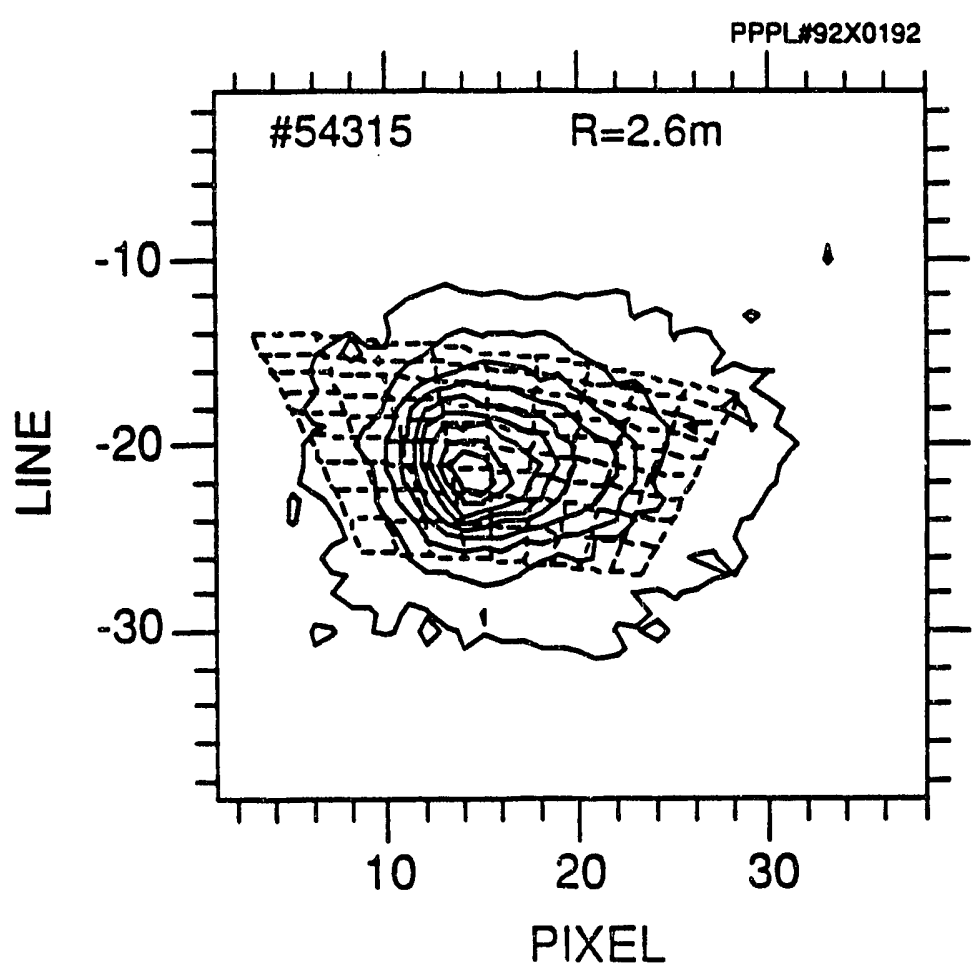

Fig. 1(b) 


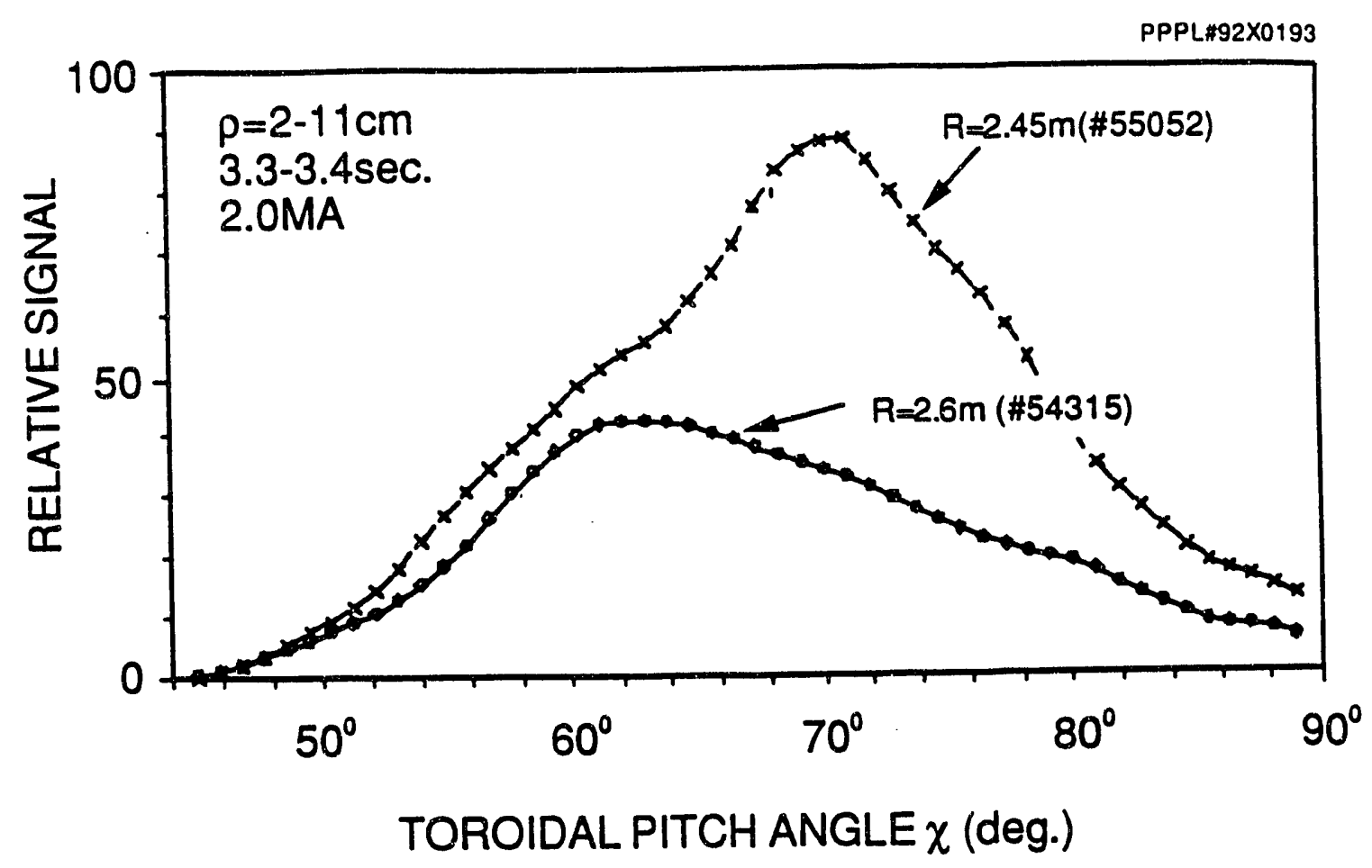

Fig. 2(a)

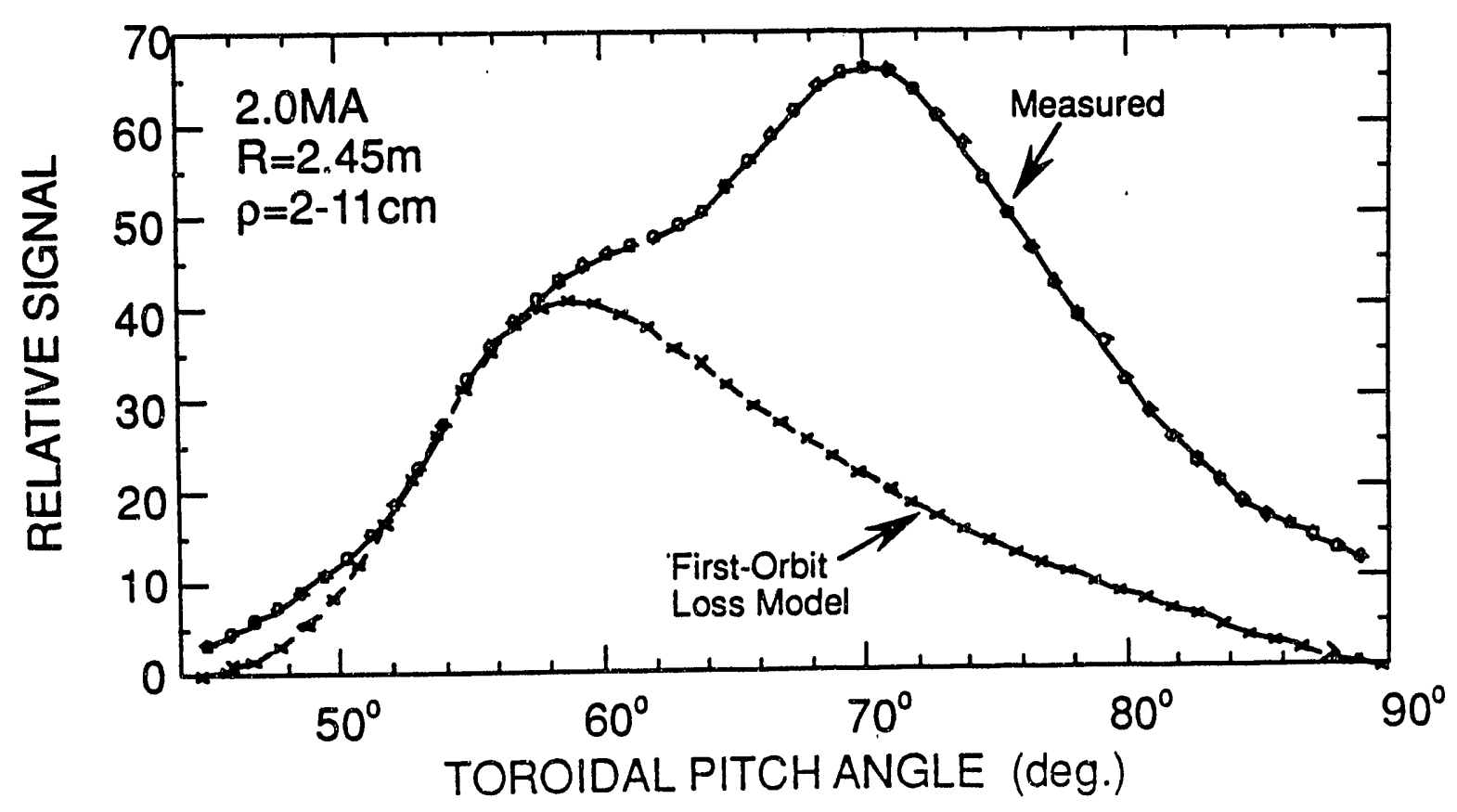

Fig. 2(b) 

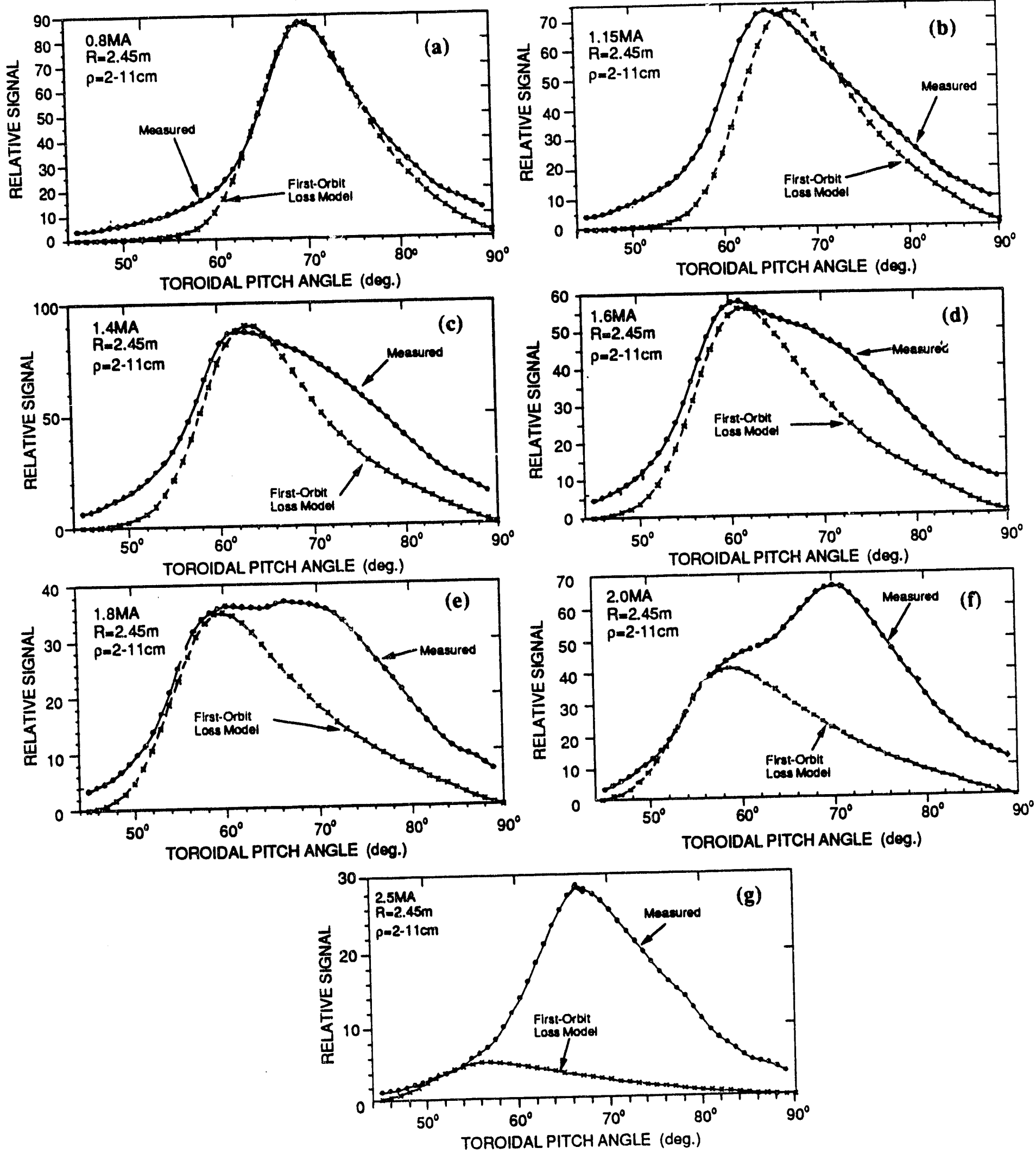

Fig.3 


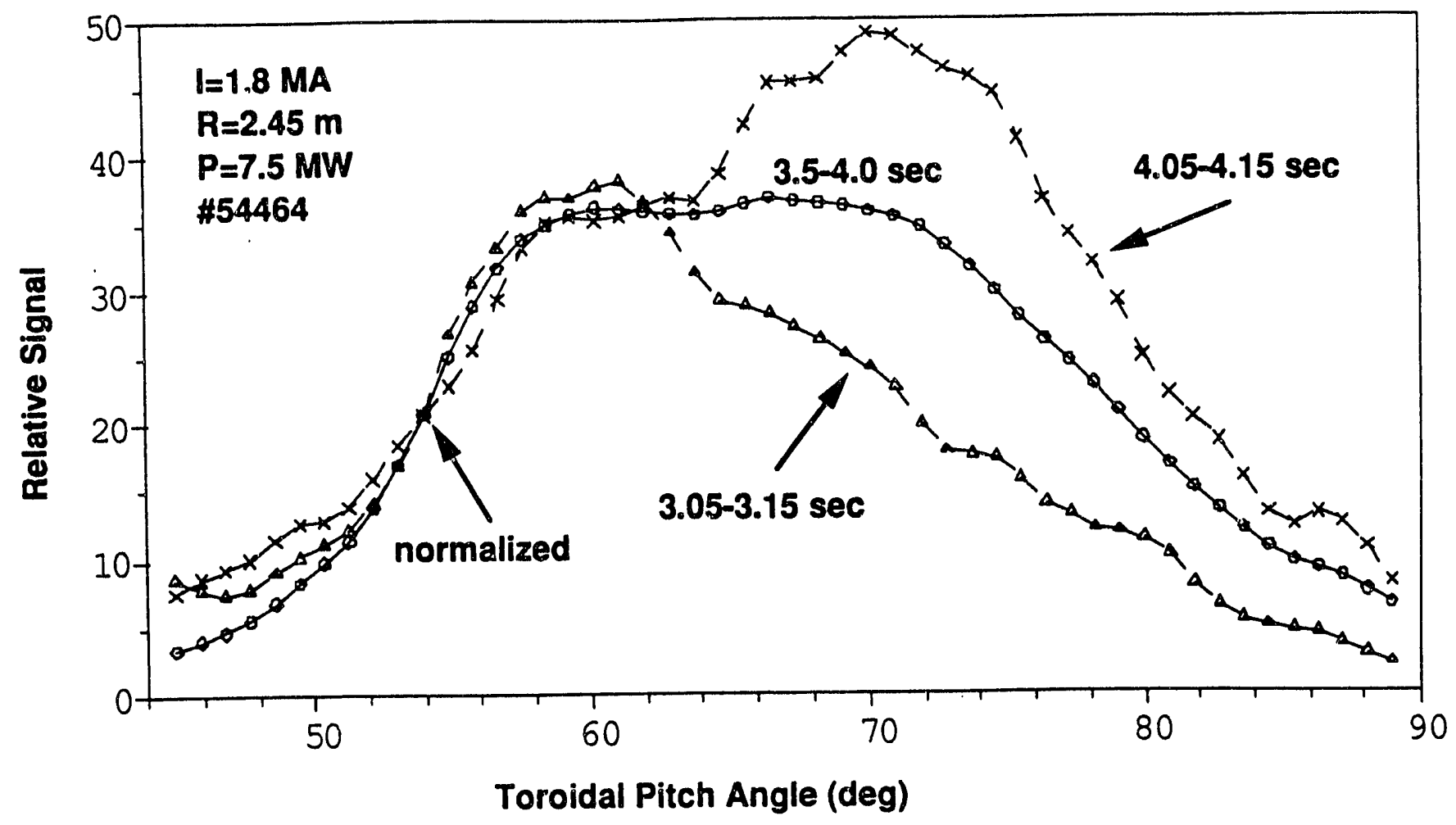

Fig. 4(a)

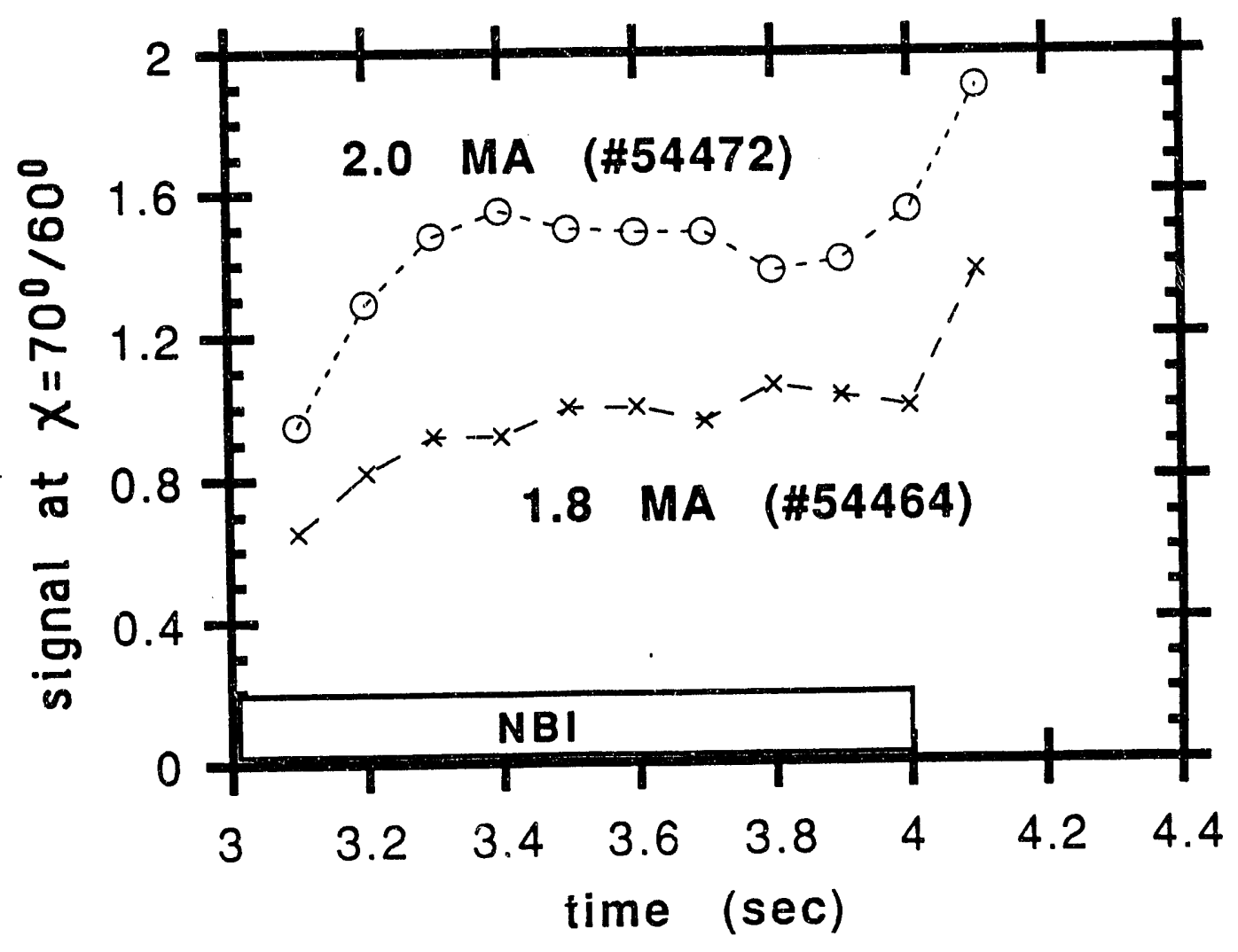

Fig. 4(b) 


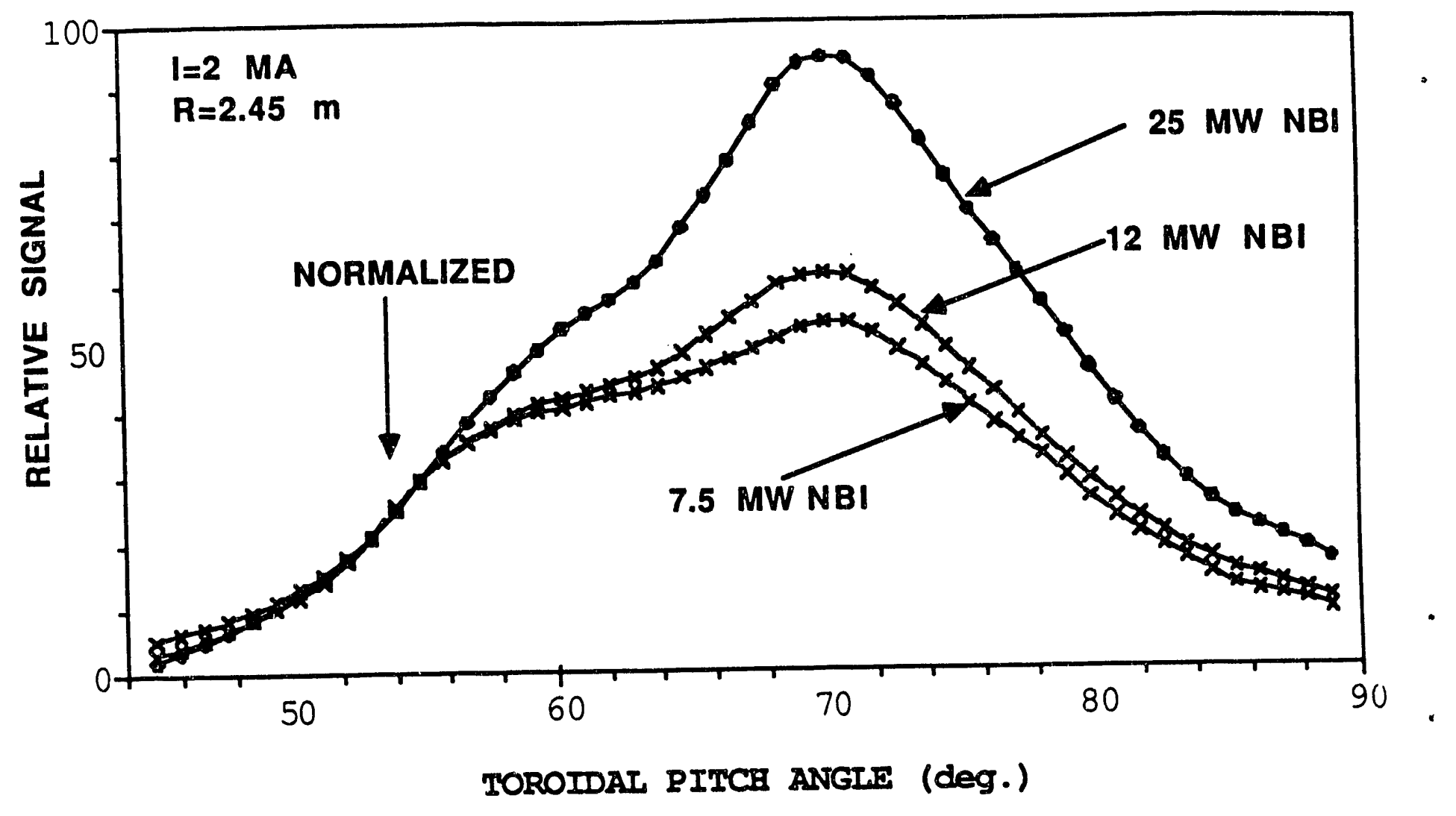

Fig. 5 


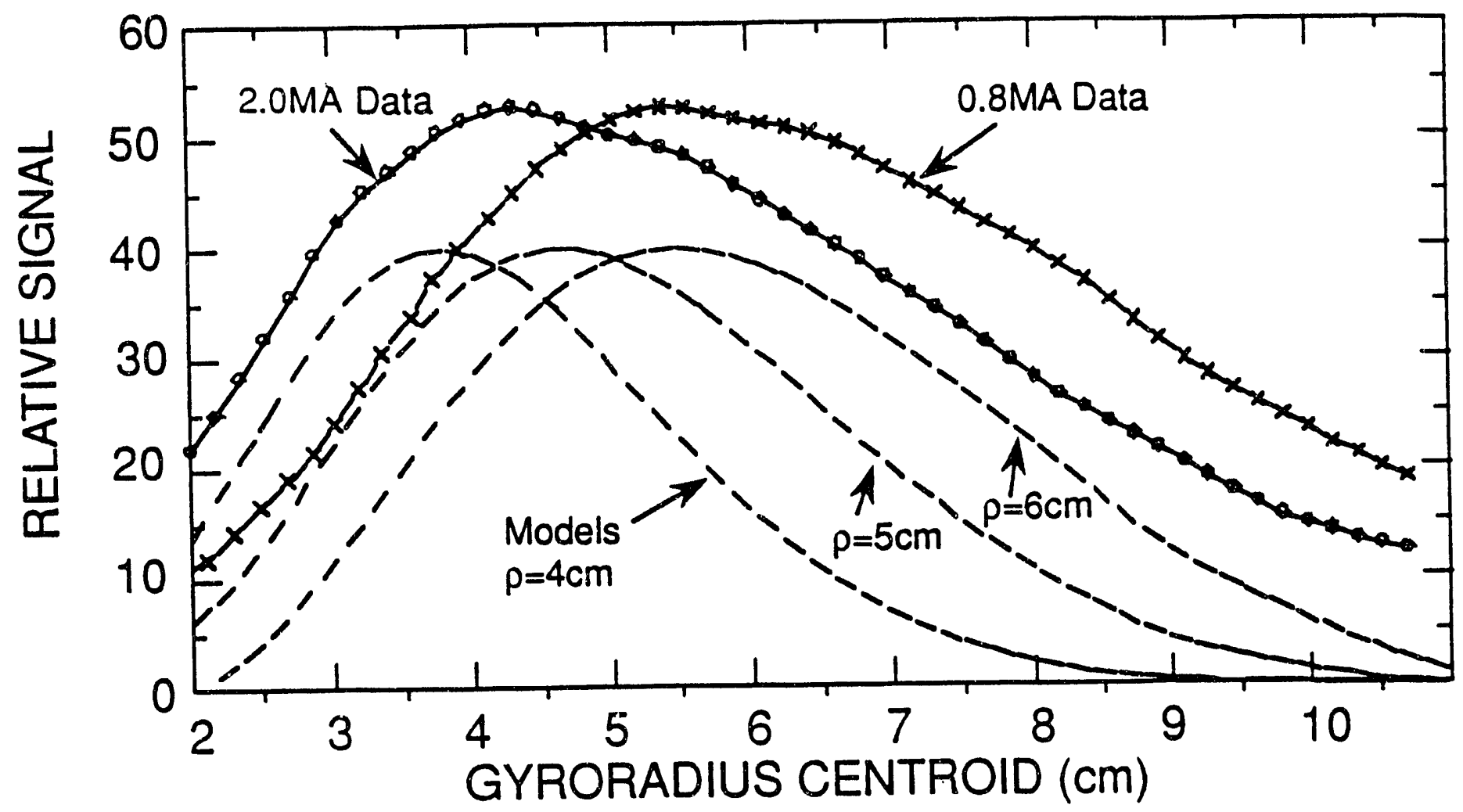

Fig. 6

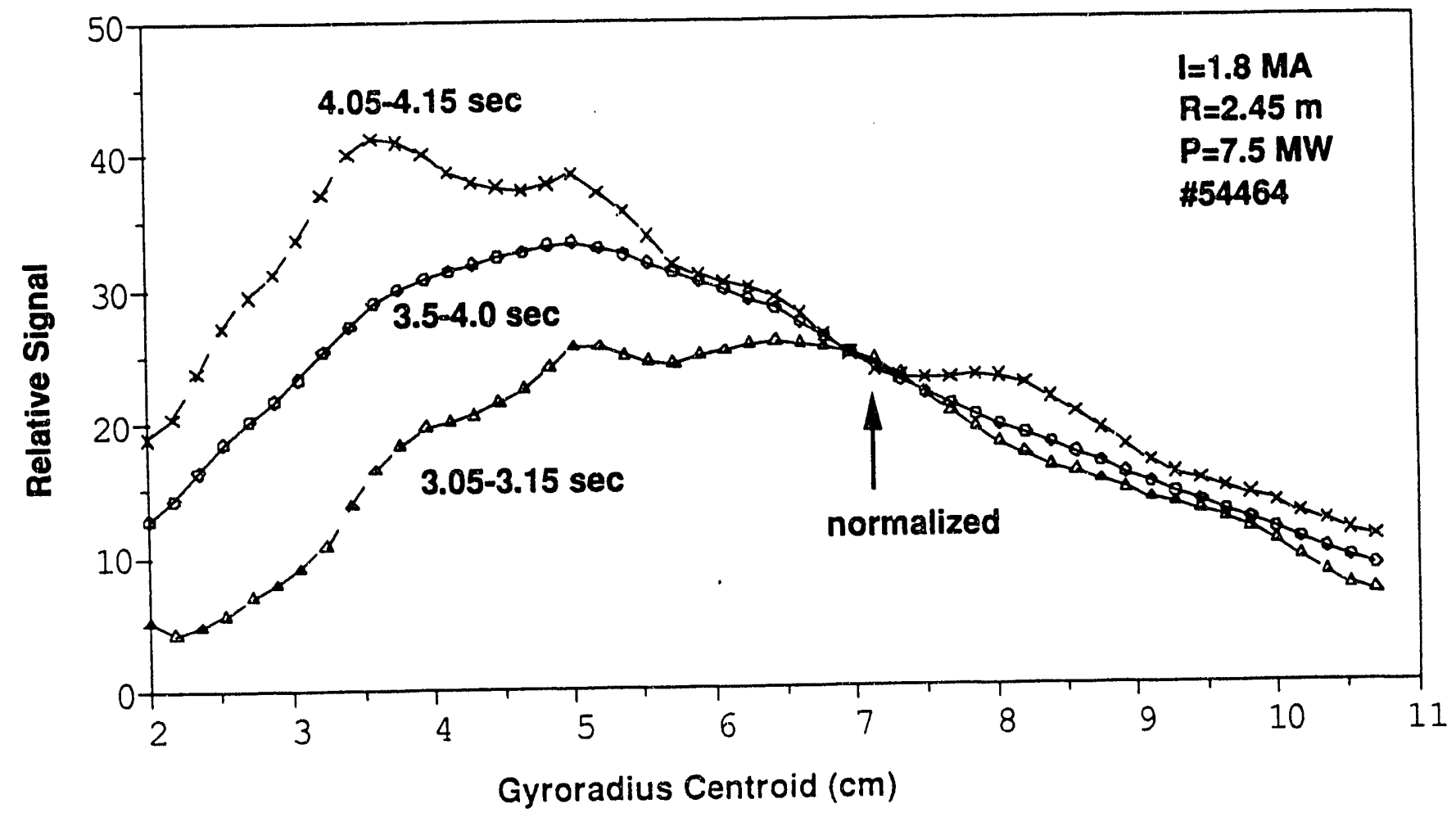

Fig. 7 


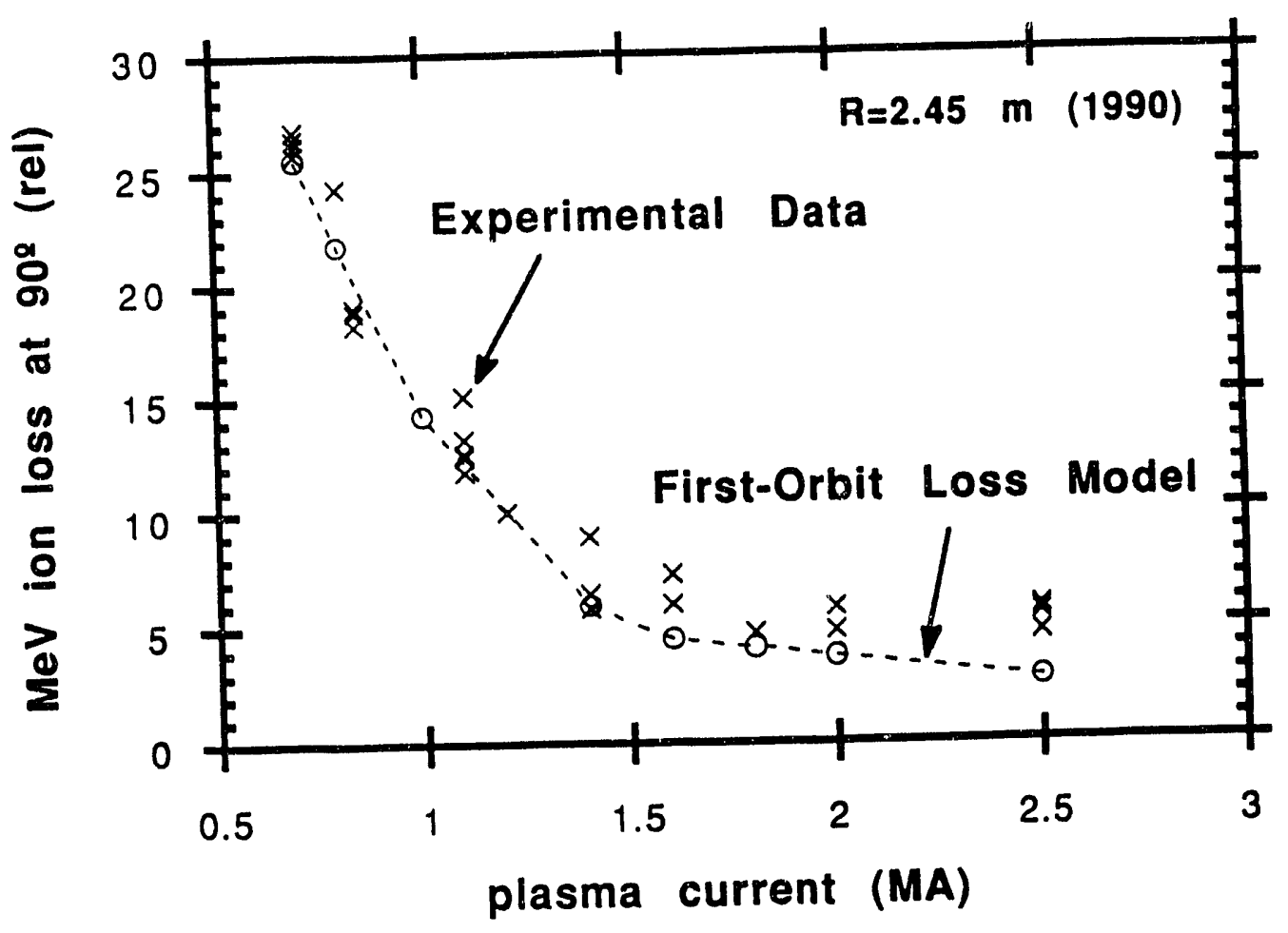

Fig. 8

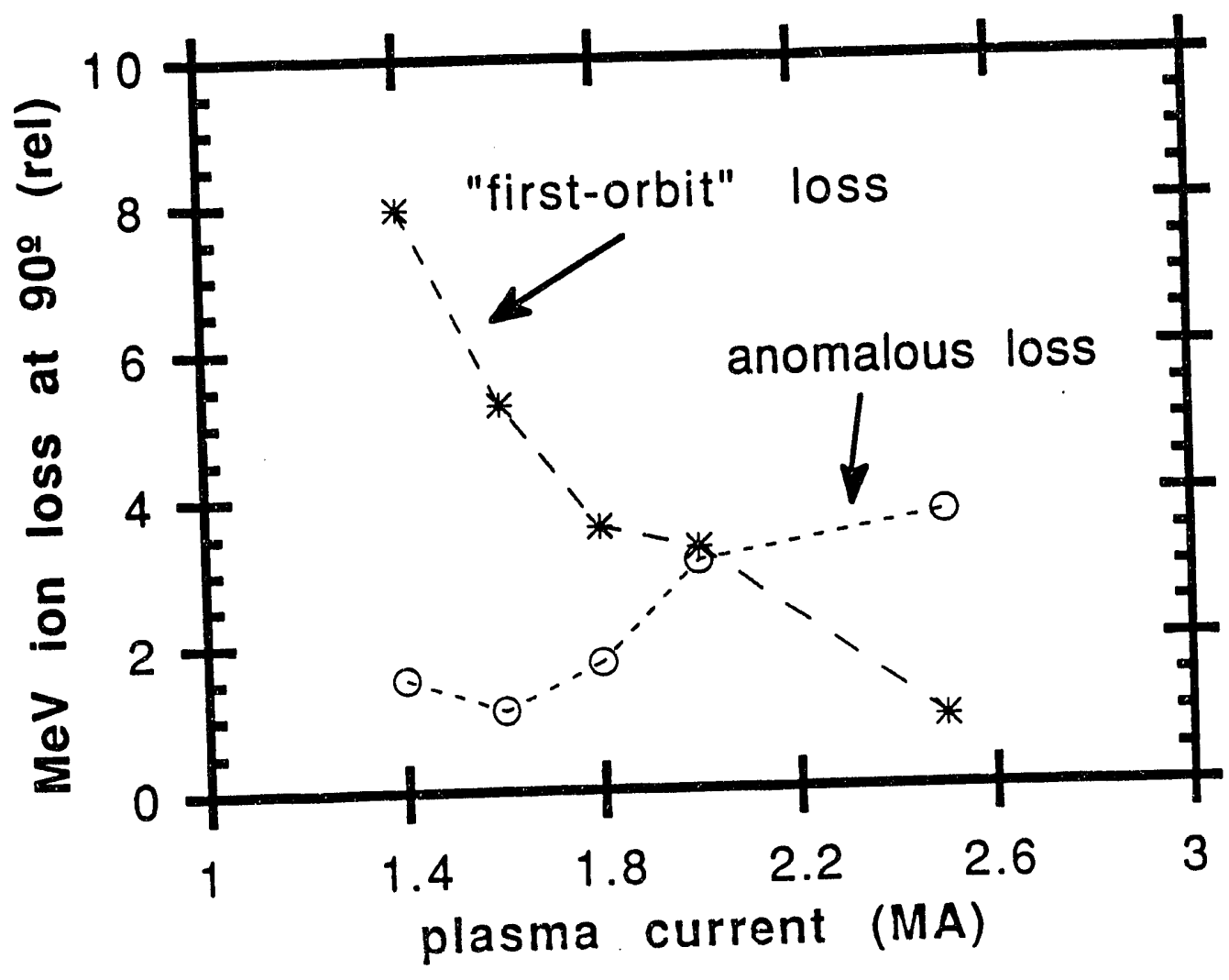

Fig. 9 


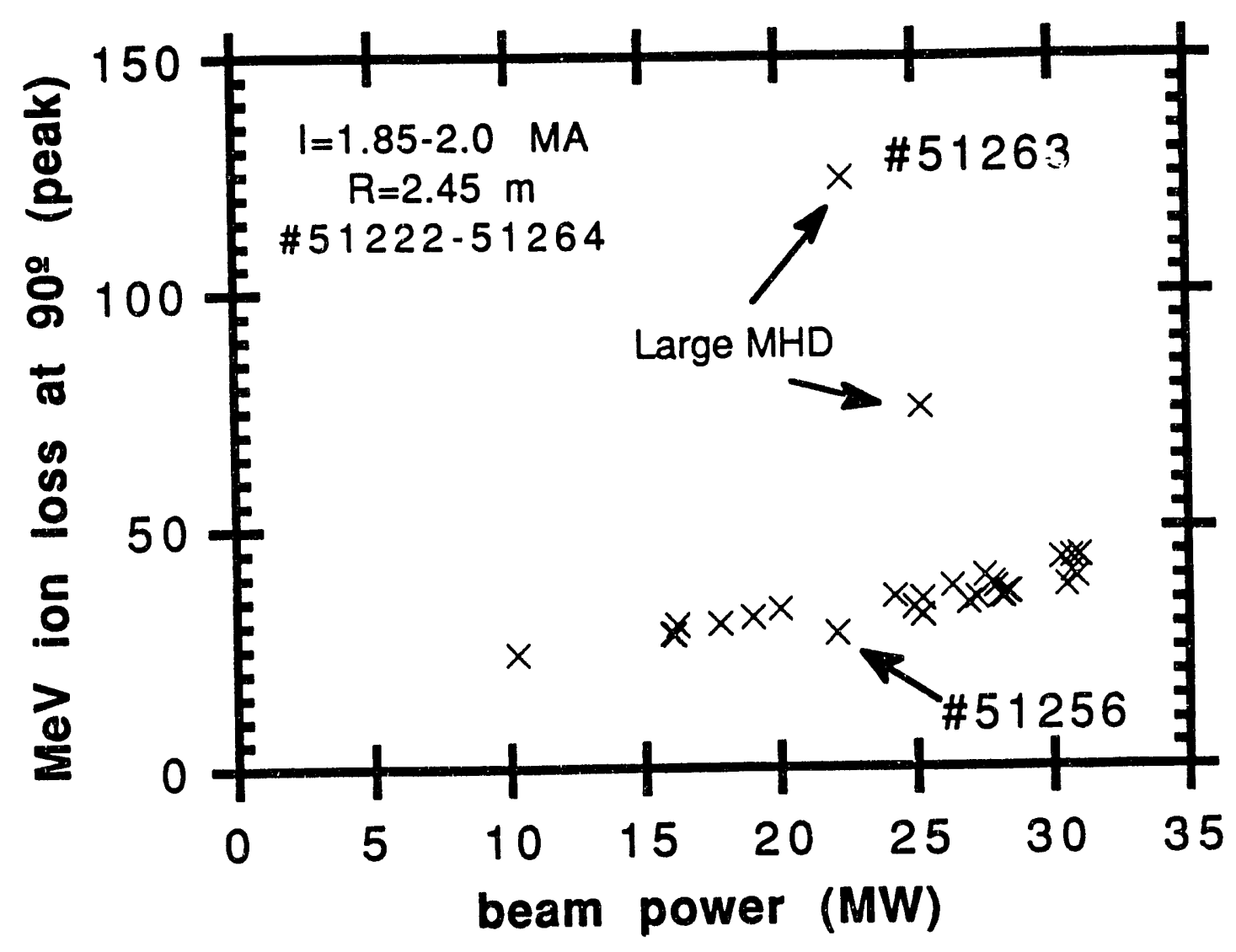

Fig. 10 


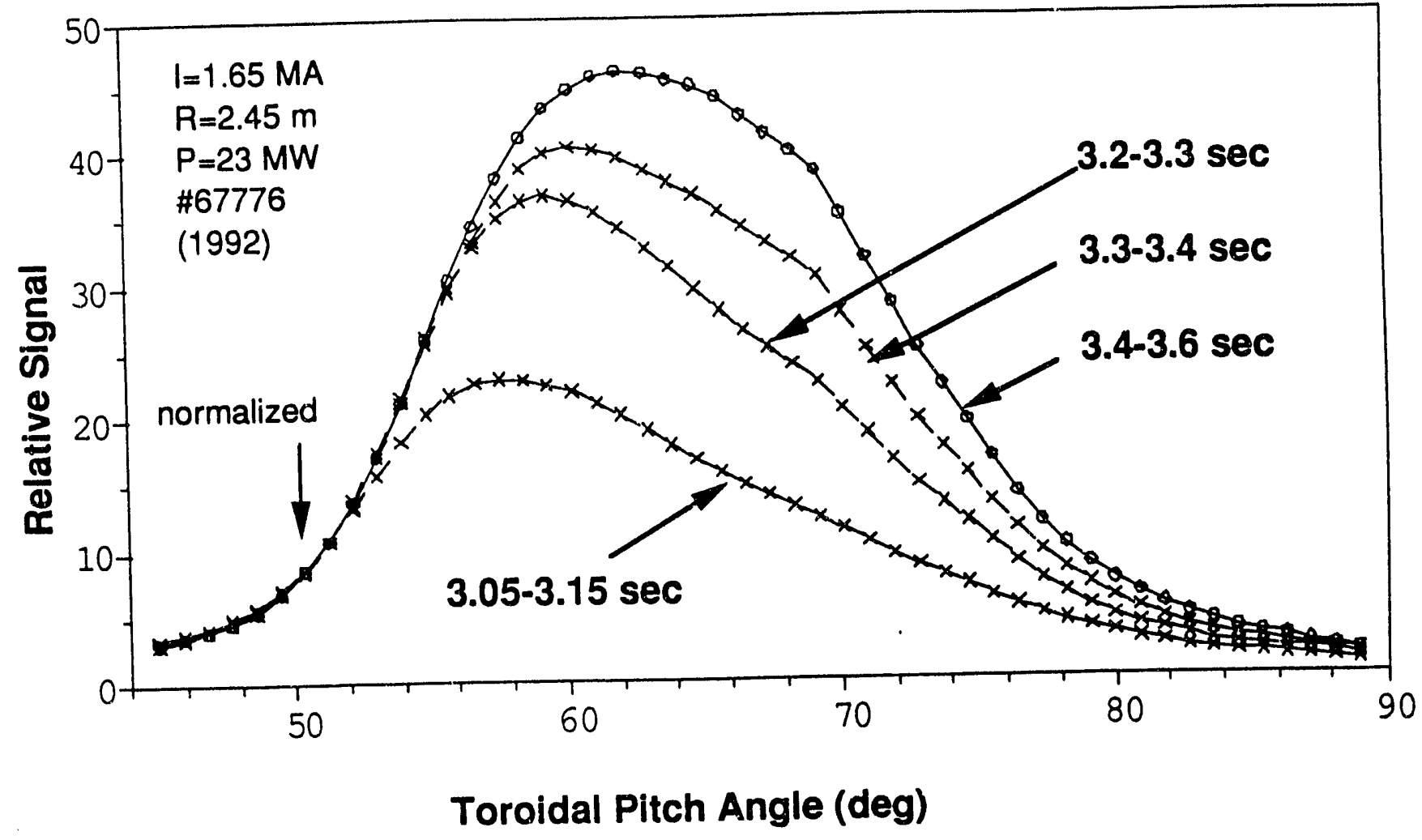

Fig. 11

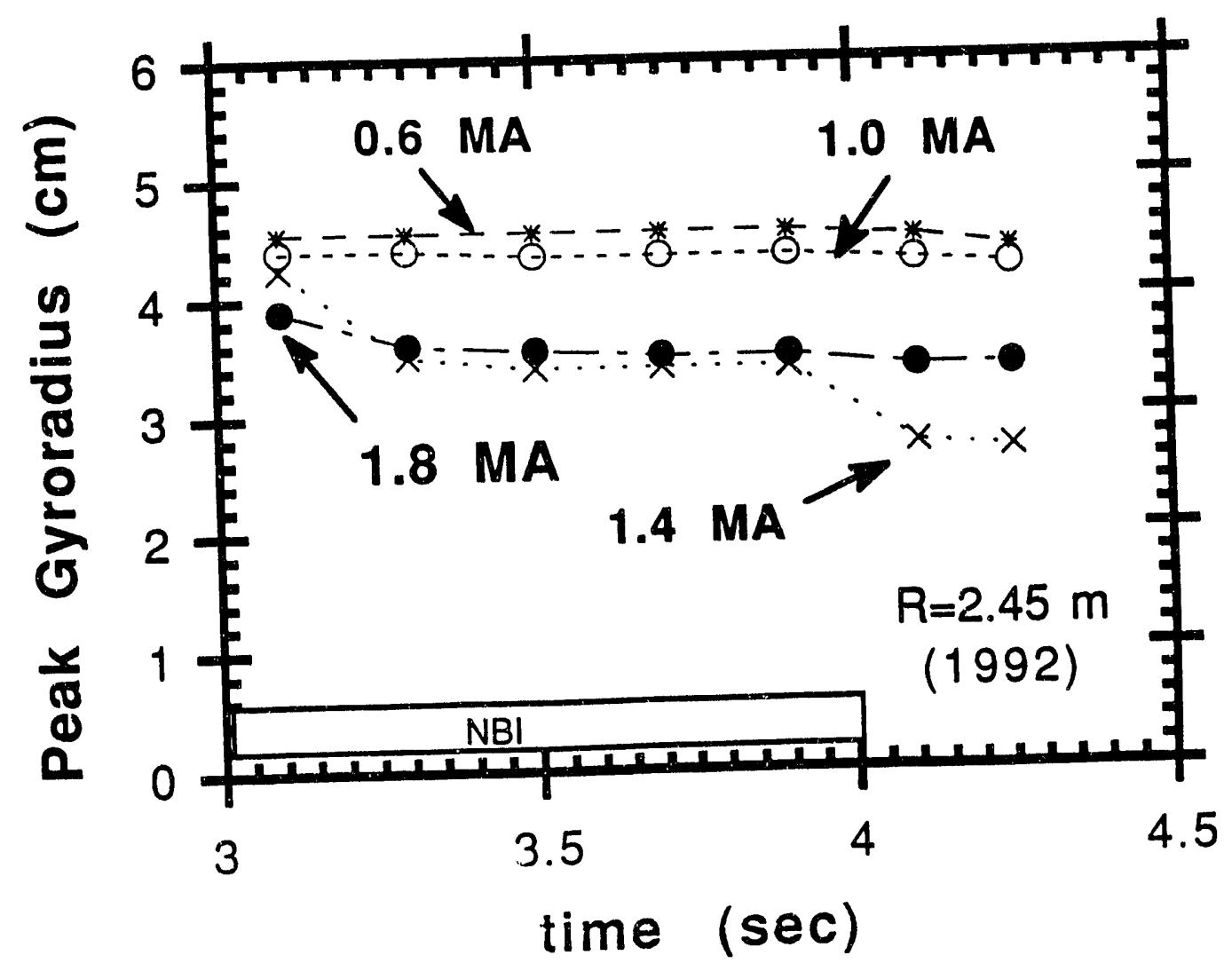

Fig. 12 


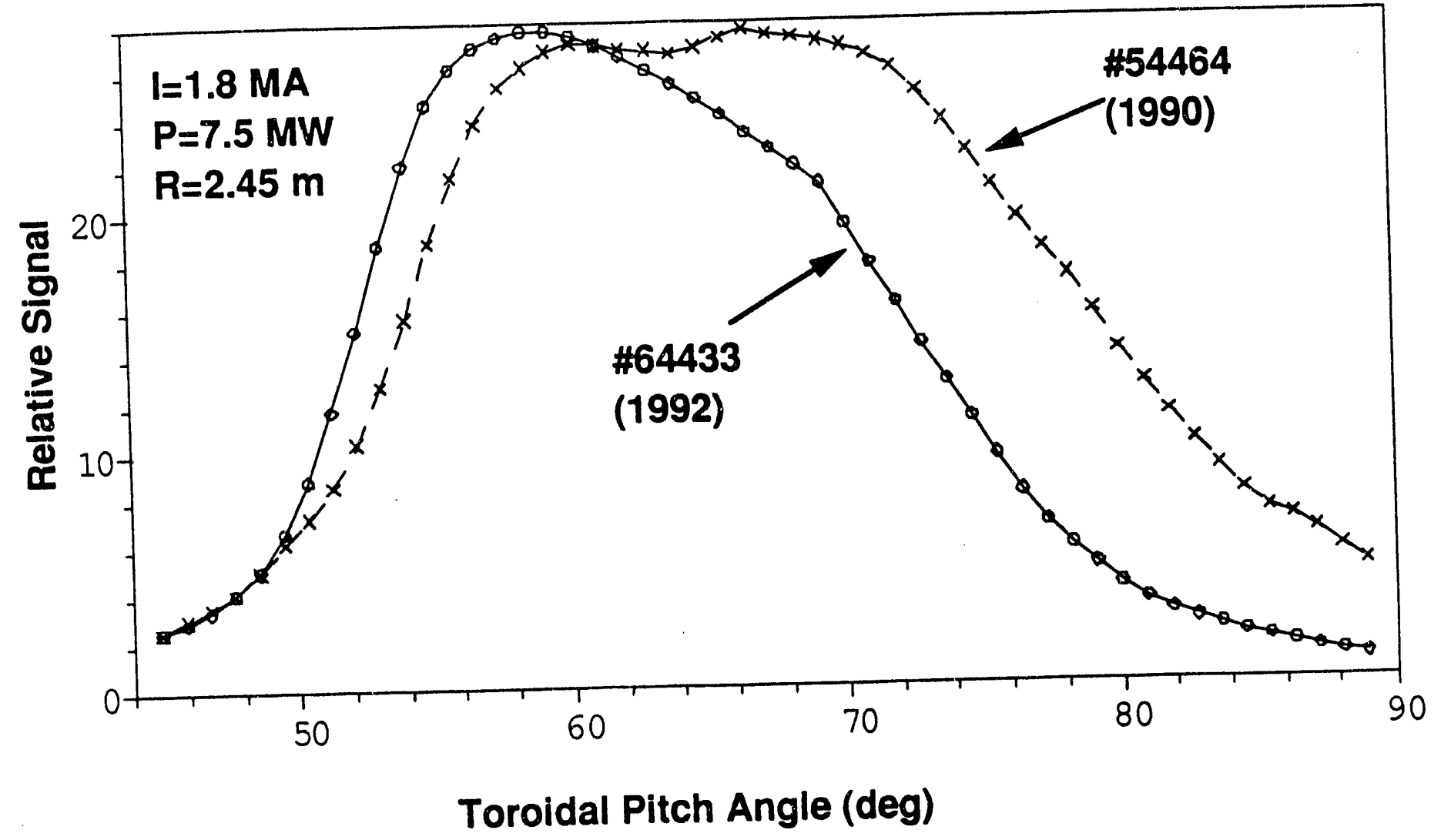

Fig. 13 

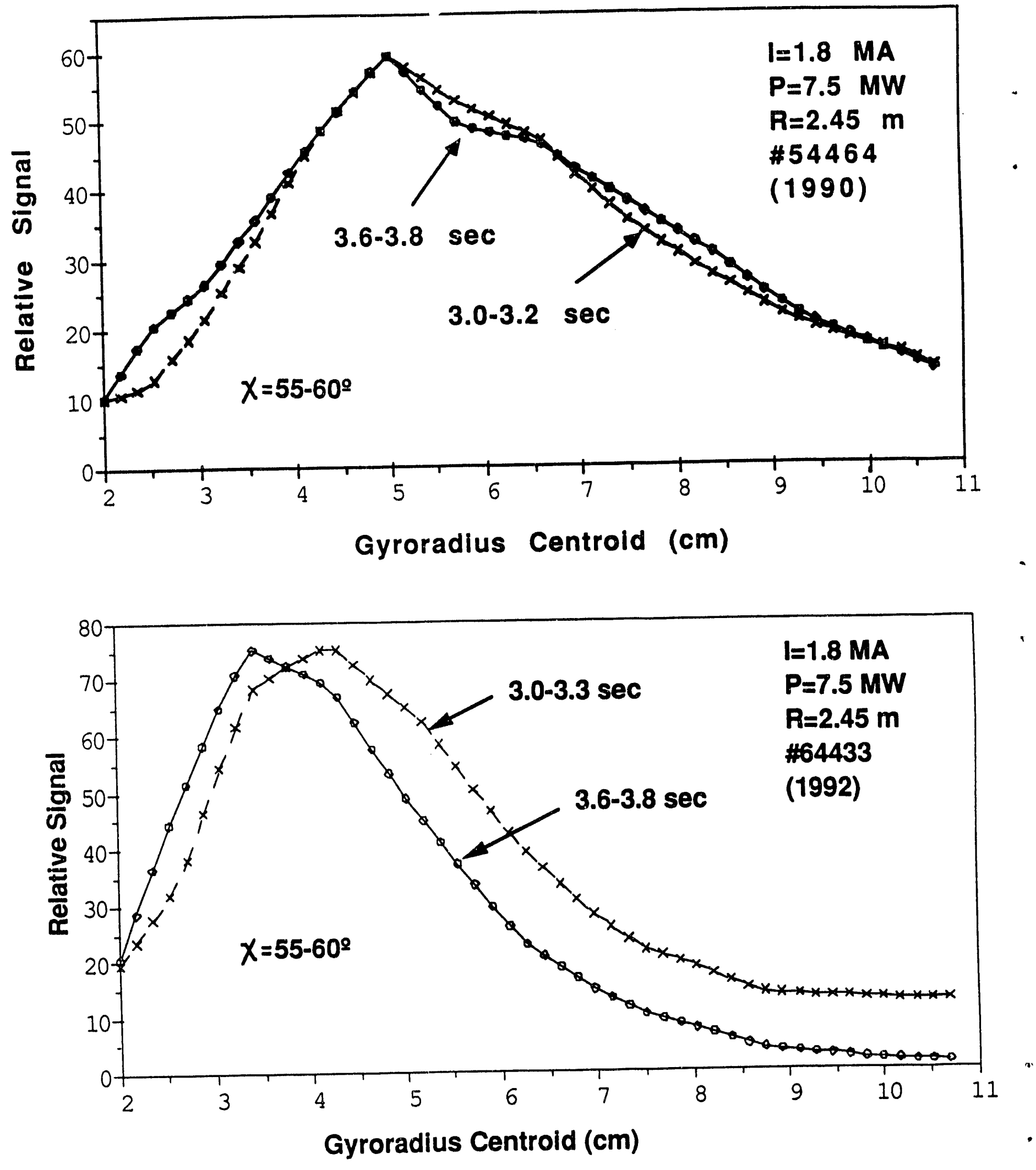

Fig. 14 


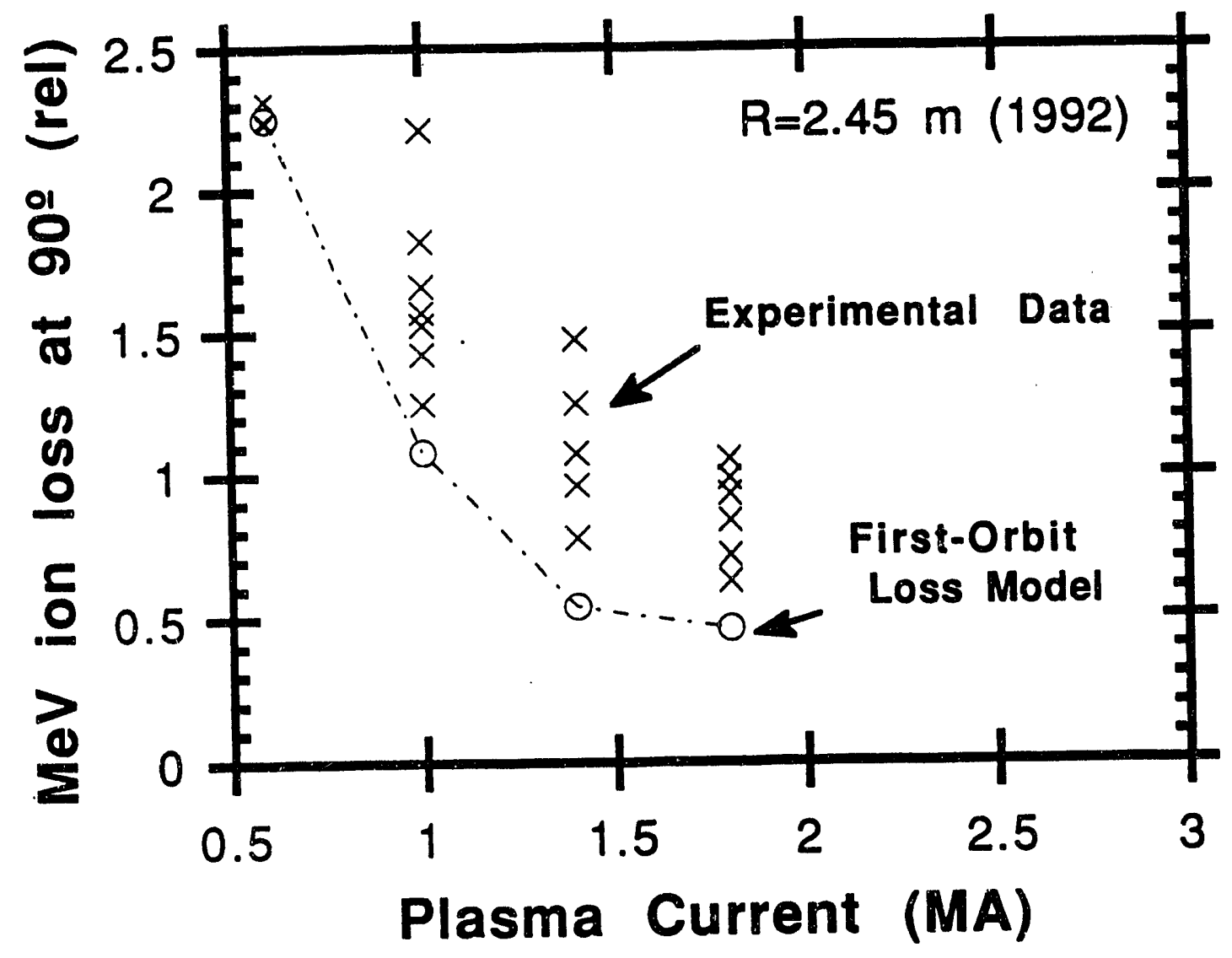

Fig. 15 

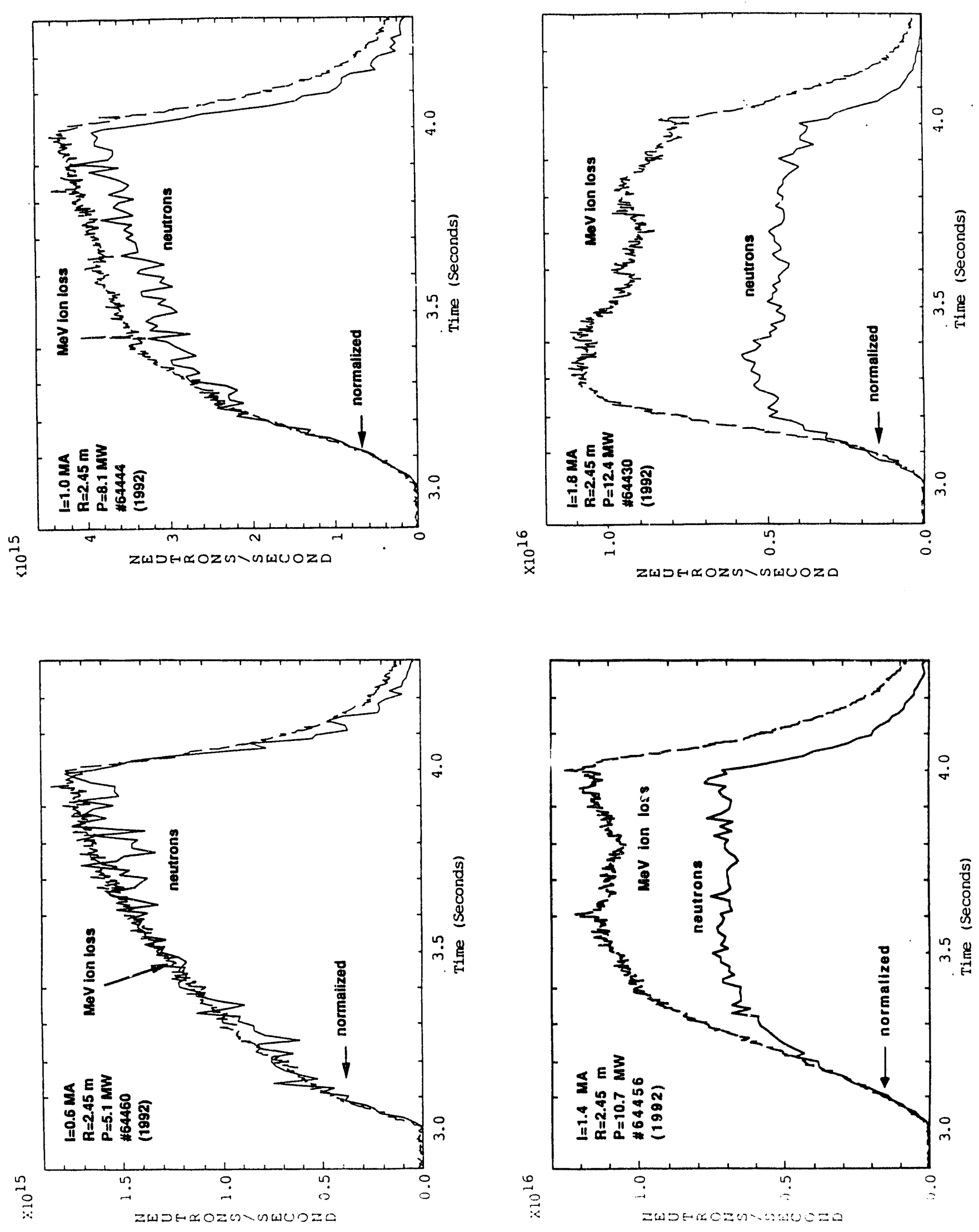

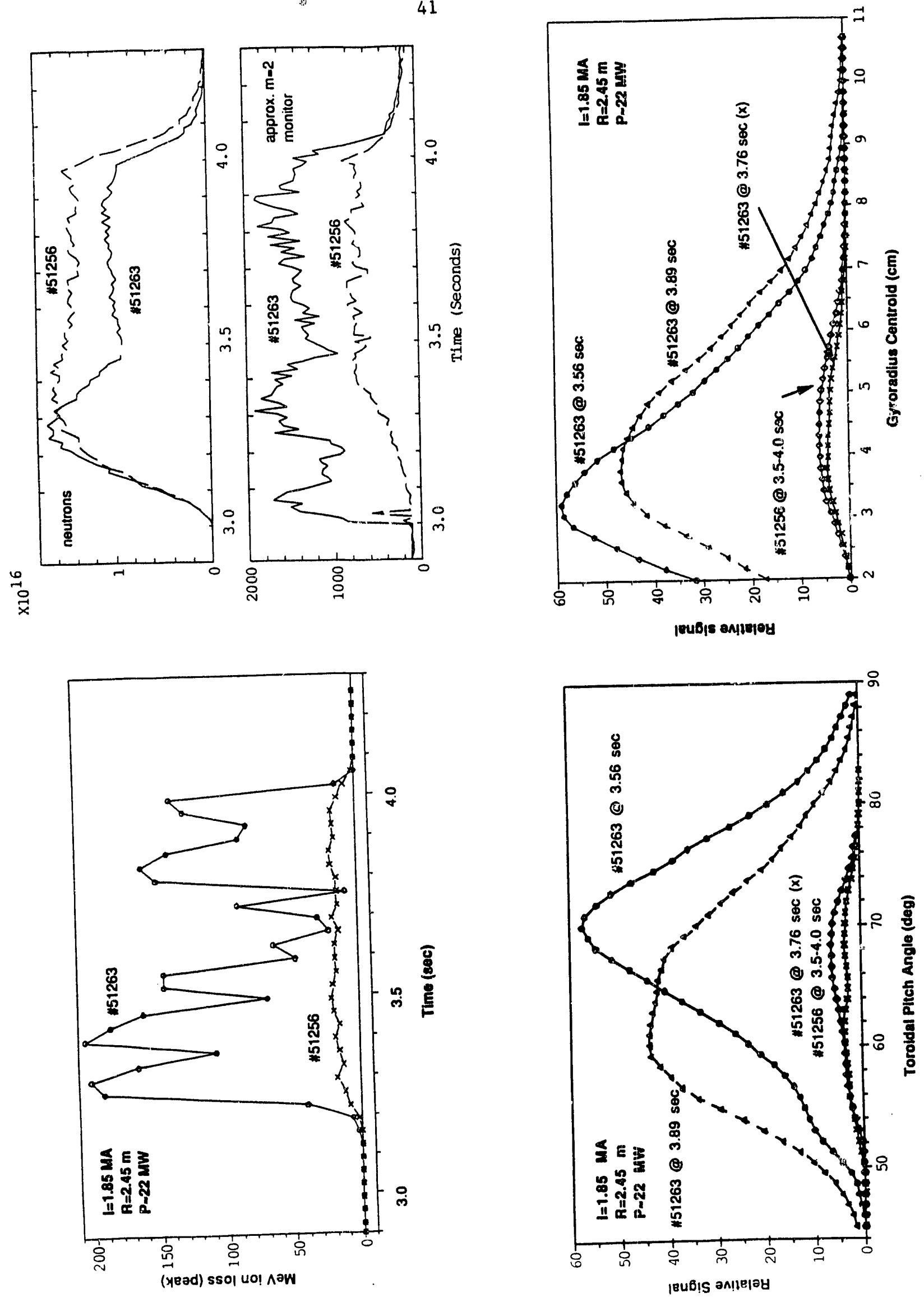

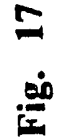



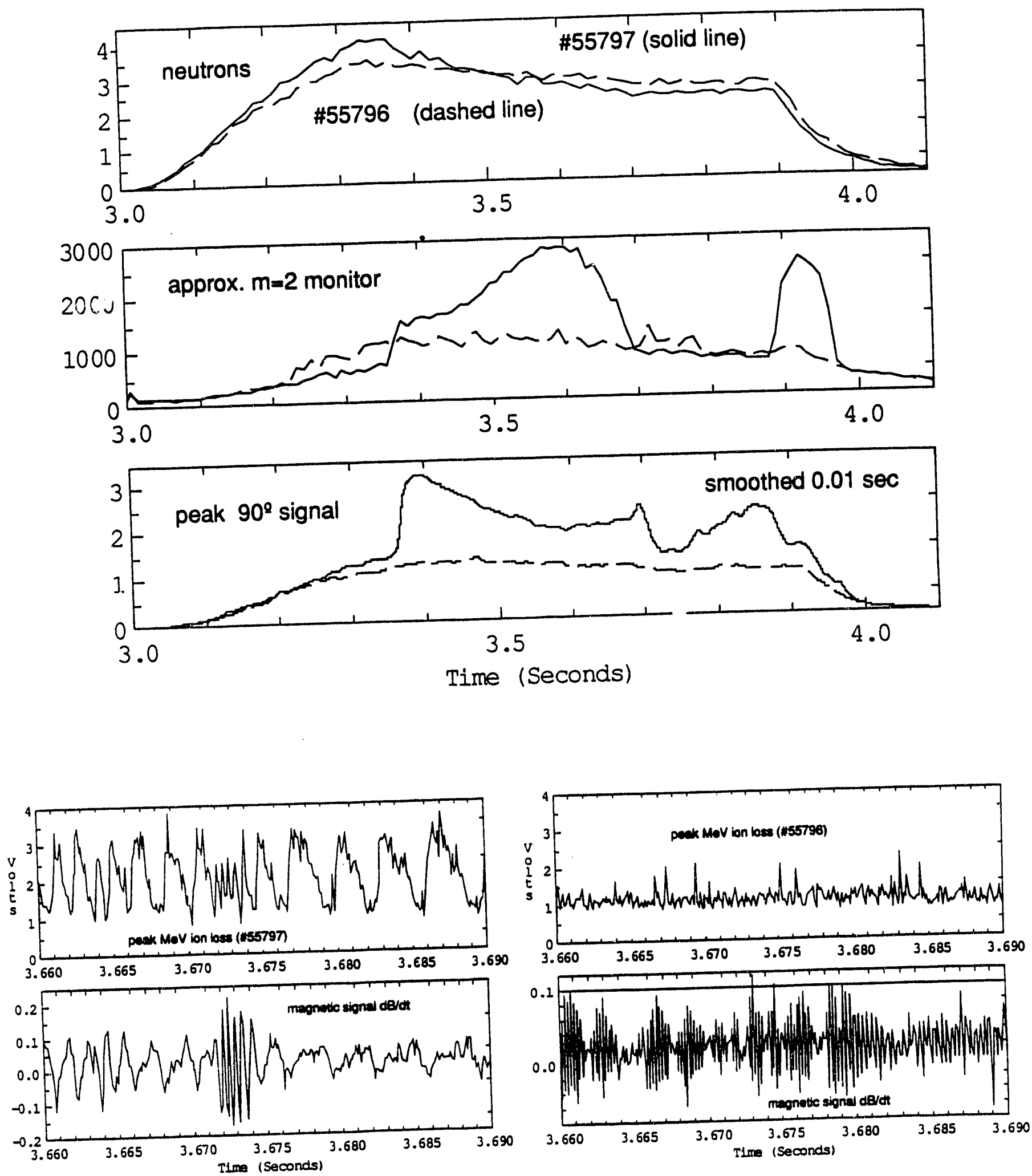

Fig. 18 

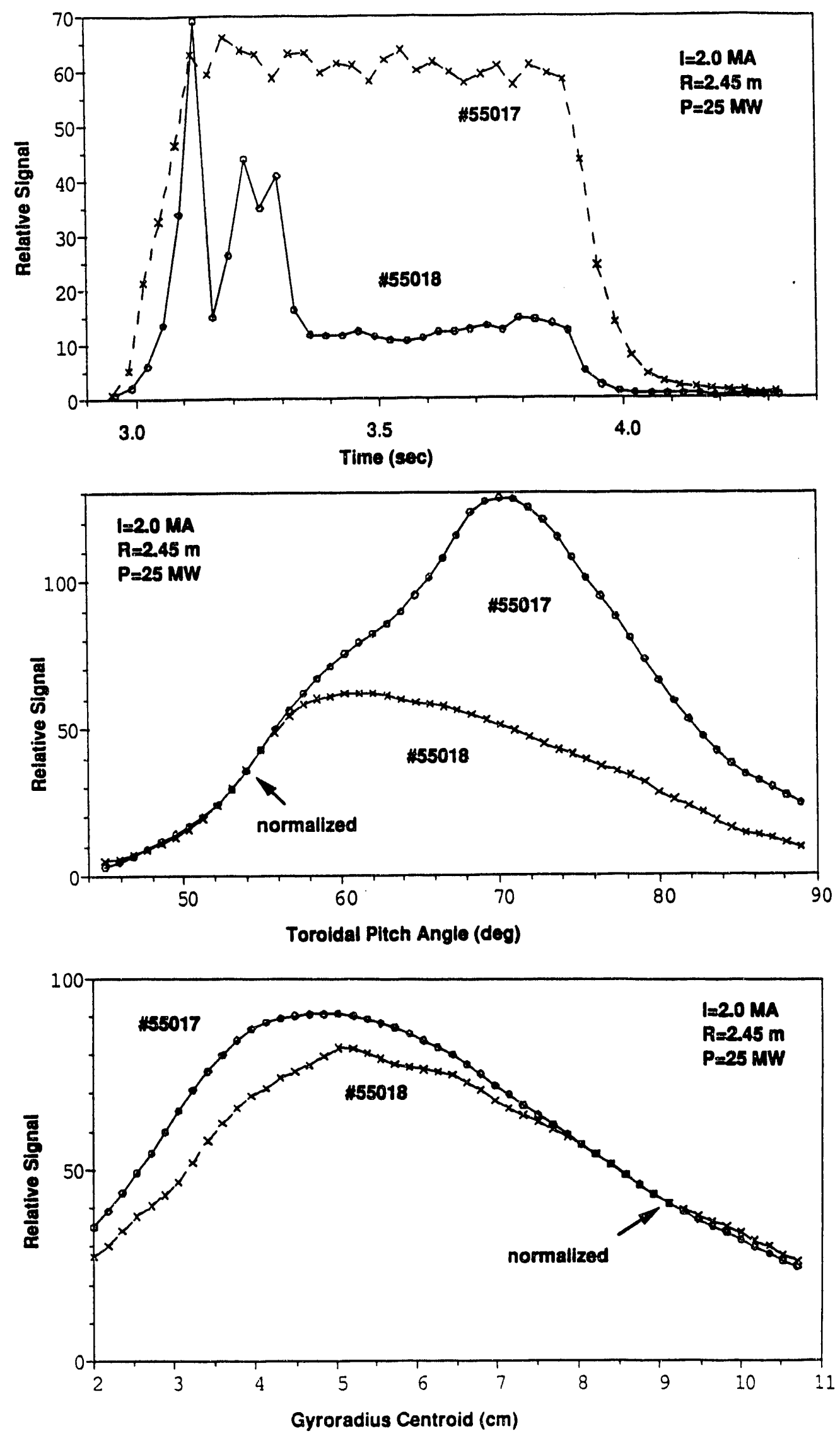

Fig. 19 


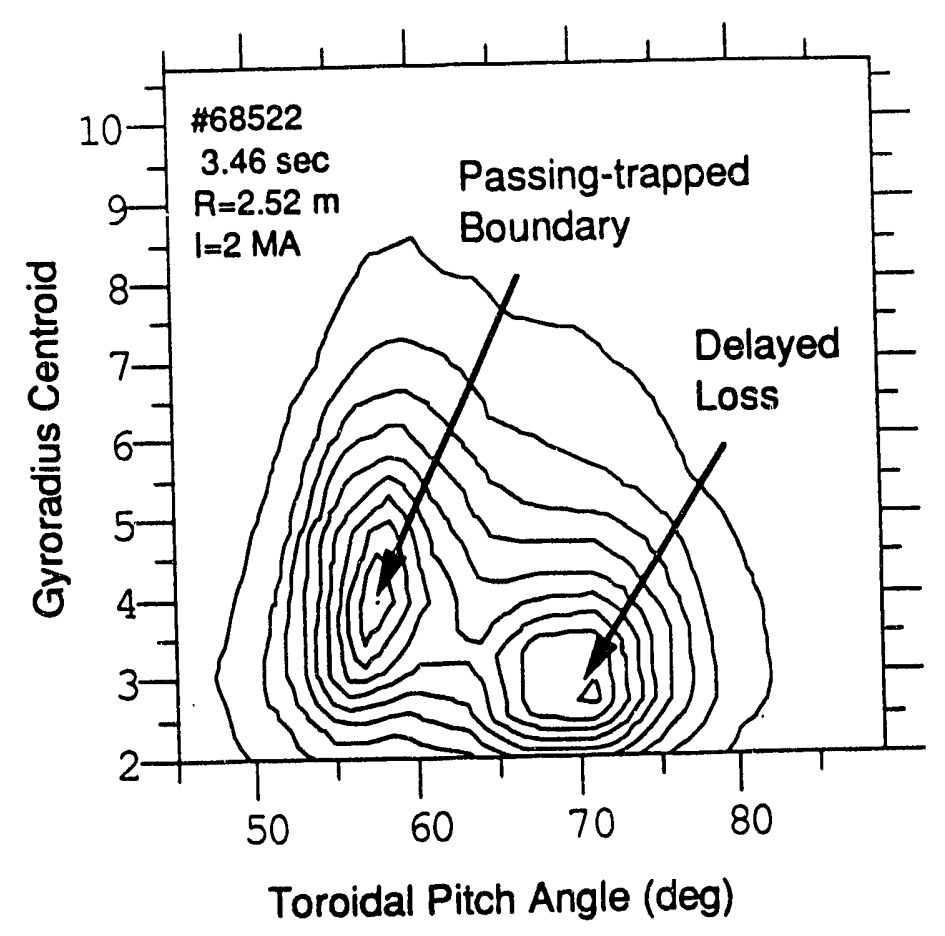

Fig. 20(a)

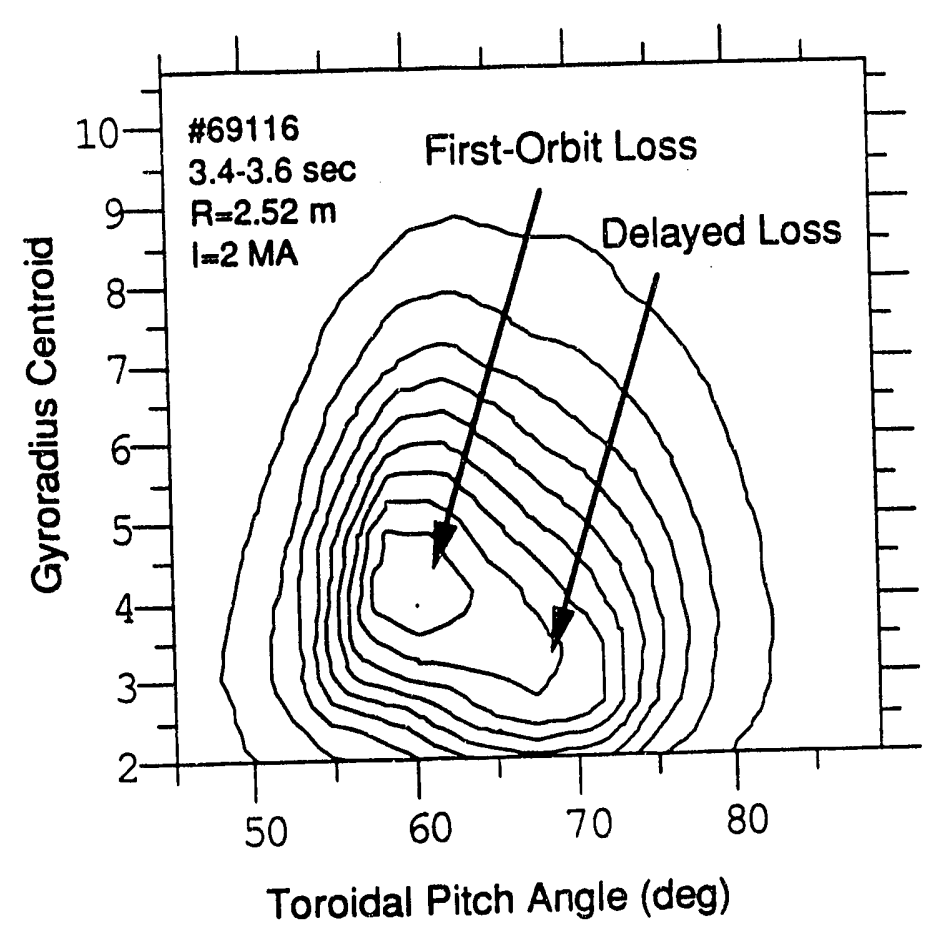

Fig. 20(b) 


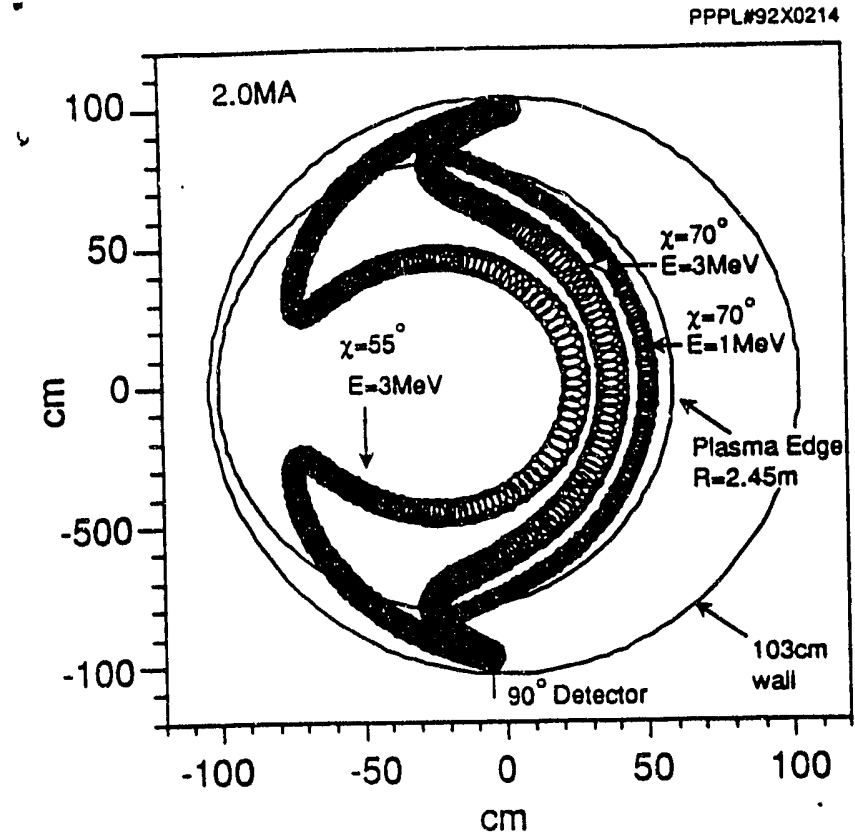

Fig. 21(a)

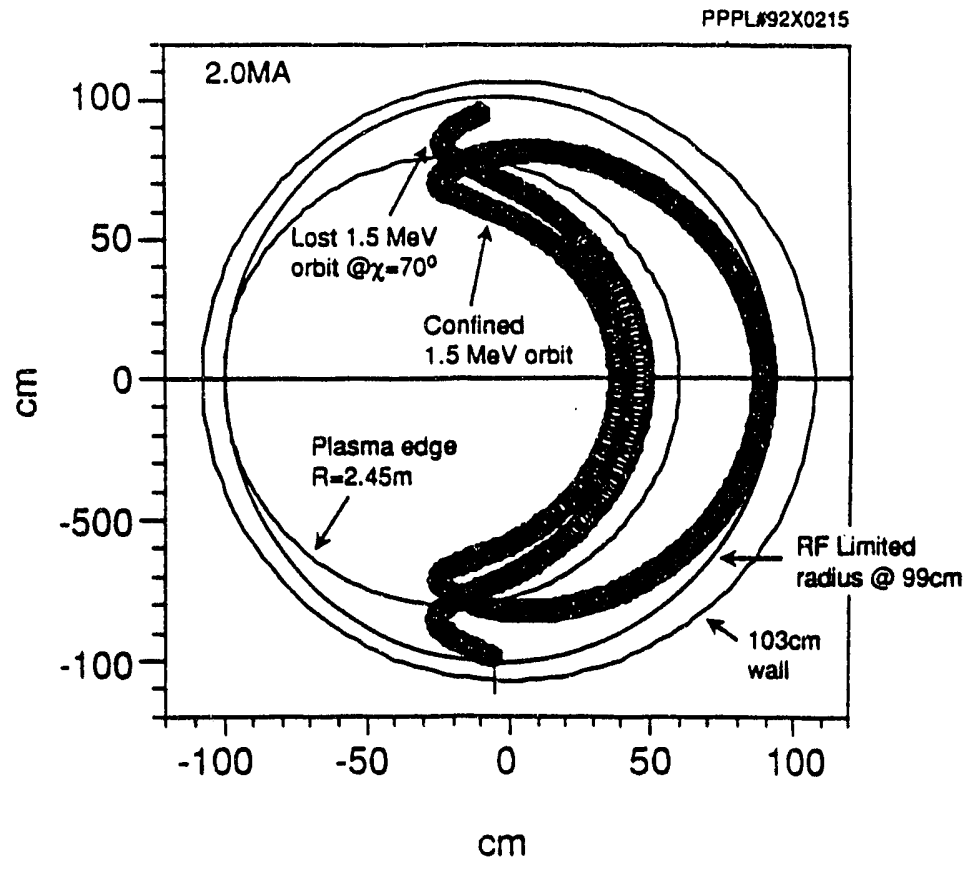

Fig. 21(b)

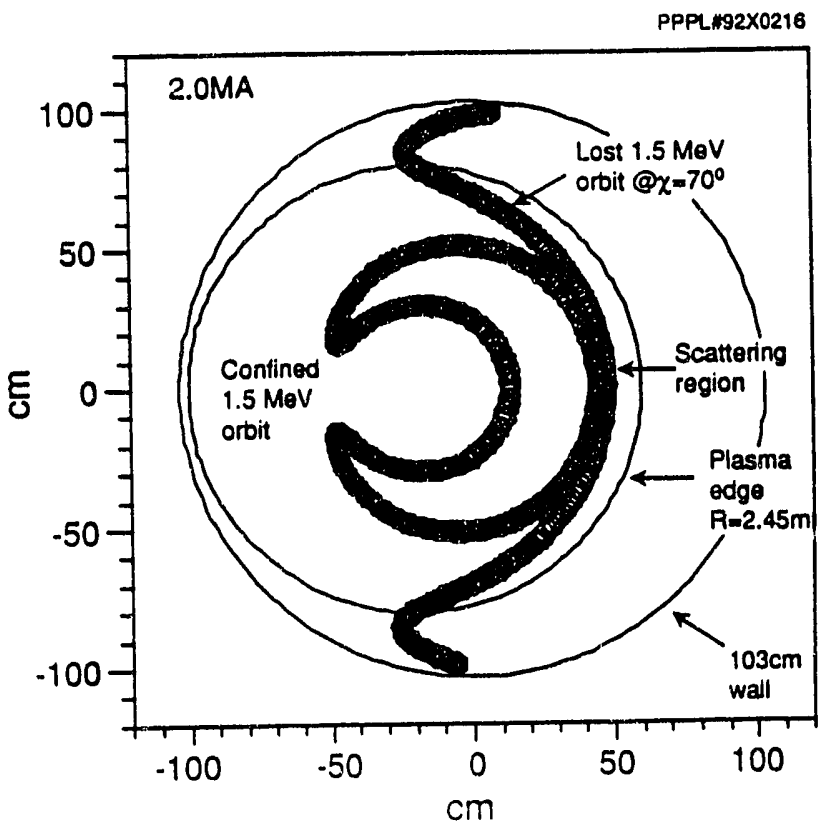

Fig. 21(c) 
Dr. F. Peoboni, Univ. of Wollongong. AUSTRALIA

Prof. M.H. Brennan, Univ. of Sydney, AUSTRALIA

Plasma Posearch Lub., Austraion Nat. Univ., AUSTRALLA

Prof. I.R. Jonos, Flindors Univ. AUSTRALLA

Prof. F. Cep, Inst for Theoretical Phyeics, AUSTRIA

Prot. M. Heindior, Insetut for Theorolischo Physik, AUSTRLA

Prof. M. Goosens, Astronomiech InetituUt, BELGIUM

Ecolo Roydo Mituiro, Lob. do Phy. Plaemas, BELGIUM

Commicaion-Europan, DG. XII-Fusion Proo., BELGIUM

Prof. R. Bouciguh, Rikeuniveritid Gent, BELGIUM

Dr. P.H. Sakencke, Inaturo Fivice, BARZLL.

Inaturto Necionet De Pecquieas Espocicio-INPE, BAAZIL

Documents Orivo, Alomic Energy of Ceneda Ld., CANADA

Dr. M.P. Bechynaki, MPB Technologises, Inc. CANADA

Or. H.M. Skergoard, Univ. of Seakatchowen, CANADA

Prof. J. Teichmem, Univ. of Montred, CANAOA

Prof. S.P. Sreenivean, Univ. of Celogry, CANADA

Prot. T.W. Johneton, INAS-Energio, CANADA

Dr. R. Batron, Contro canadien de husion meanstique, CANADA

Dr. C.R. James., Univ. of Nberte, CANADA

Dr. P. Lukde, Komonaththo Univerexita, CZECHOSLOVAKIA

The Librarian, Culnam Leboratory, ENGLAND

Librery, R61, Ruthertord Applecion Laboratory. ENGLAND

Mrs. S.A. Hulchireon, JET Librery, ENGLAND

Dr. S.C. Shema, Univ. of South Pecific, FWII ISLANDS

P. Muthonon, Univ. of Hedeinki, FINLAND

Prot. M.N. Buseac, Ecols Pobyectrique., FPANCE

C. Mourat, Leb. de Phycizue des Mitieux lonieds, FRANCE

J. Radht, CENCADARACHE - Bat 506, FRANCE

Prot. E. Economou, Univ. of Crow, GREECE

Ms. C. Rinni, Univ. of loemina, GAEECE

Dr. T. Mud, Acadermy Ebliogrephic Ser., HONG KONG

Preprint Librey, Mungarian Acadormy of Sci., HUNGAPY

Dr. B. Descupta, Saha inst of Nuctere Phycics, INDIA

Dr. P. Kaw, Inet. for Pleema Research, INDUA

Dr. P. Rosenwe, lened inst of Tectnology, ISPAEL

Librerien, Internationd Cember for Theo Physica, ITALY

Miss C. Do Palo, Askociazione EURATOMENEA, ITALY

Or. G. Groses, Istiuw di Firica ded Plasma, ITALY

Prof. G. Rostengni, letituso Gas lonizzadi Del Cnr, ITALY

Dr. H. Yamato, Tochiba Res a Dovel Center, JAPAN
Prot. I. Kawakemi, Hirochima Univ., JAPAN

Prof. K. Nishikewa, Hiroshima Univ., JAPAN

Diroctor, Jepen Atomic Enorgy Rosoarch inst, JAPAN

Prof. S. Ioh, Kyuahu Uniry., JAPAN

Rosararch Into. Ctr., National Instit. for Fusion Science. JAPAN

Prof. S. Tenaka, Kyolo Univ., JAPAN

Librey, Kyow Univ., JAPAN

Prot. N. Inow, Univ, of Tokyo, LAPAN

Sacretany, Plesma Saction, Elactrowchniced Lab., JAPAN

S. Mori, Technied Advieor, JAEPI, JAPAN

Dr. O. Mud, Kumenoto het of Technology, JAPAN

J. Ayron-Sook, Korea Abpric Enorgy Roserech Inet, KOAEA

D.I. Choi, Thw Kore Adv. Inst. of Sai. \& Tech., KOREA

Prot. B.S. Liby, Univ. of Weikato, NEW ZEALAND

Inat of Phycics, Chinces Acad Sa PEOPLE'S AEP. OF CHINA

Librey, Inat of Plasma Physics, PEOPLE'S AEP. OF CHINA

Tainghua Univ. Librery, PEOPLE'S REPUBLC OF CHINA

Z. Li, S.W. Inet Phyeica, PEOPLE'S REPUBUC OF CHINA

Prol. J.A.C. Cabre, insiuto Superior Tecnico, PORTUGAL

Dr. O. Porus, Al I CUza Univ., ROManIA

Dr. J. do Viliers, Fusion Sudies, AEC, S. AFAICA

Prot. M.A. Hewborg. Univ. of Natw, S. AFAICA

Prot. O.E. Km, Poheng Inat. of Sa. \& Tech., SO. KOREA

Prof. C.I.E.M.A.T, Fusion Oivision Library, SPAIN

Dr. L Stonfo, Univ. of UMEA, SWEDEN

Lorey, Royd inct of Technology, SWEDEN

Prof. H. Whationsen, Chadmars Univ. of Tech., SWEDEN

Centro Phys. Das Phemas, Ecolo Poyrech, SWITZERLAND

Eroliotheak, Inat. Voor Plaena-Fyeica, THE NETHERLANDS

Aest. Prot. Dr. S. Codir, Midet Esat Tech. Univ., TURKEY

Dr. VA. Cuthith, Sci. Res. Inet Eloctrophys.I Apparatus, USSR

Dr. D.D. Pyutov, Siberian Brench of Acadomy of Sa., USSR

Dr. GA. Eliewer, I.V. Kurchutov Inst, USSA

Librerion, The Ukr.SSA Acedemy of Scionces, USSR

Dr. LM. Kowizhnyth, Inct. of Ganerd Phyzica, USSR

Kemforechungeanlaps GmbH, Zentrebibliothek, W. GERMANY Bibliothek, Inat. For Plasmatorichung, W. GEAMANY

Prof. K. Schinder, Auhr-Univereitu Bochum, W. GERMANY

Dr. F. Wagner, (ASOEX), Max-Planck-Institut, W. GERMANY

Librarian, Max-Plenck-Institur, W. GERMANY

Prof. R.K. Janev, Inst of Physica, YUGosLaVIA 

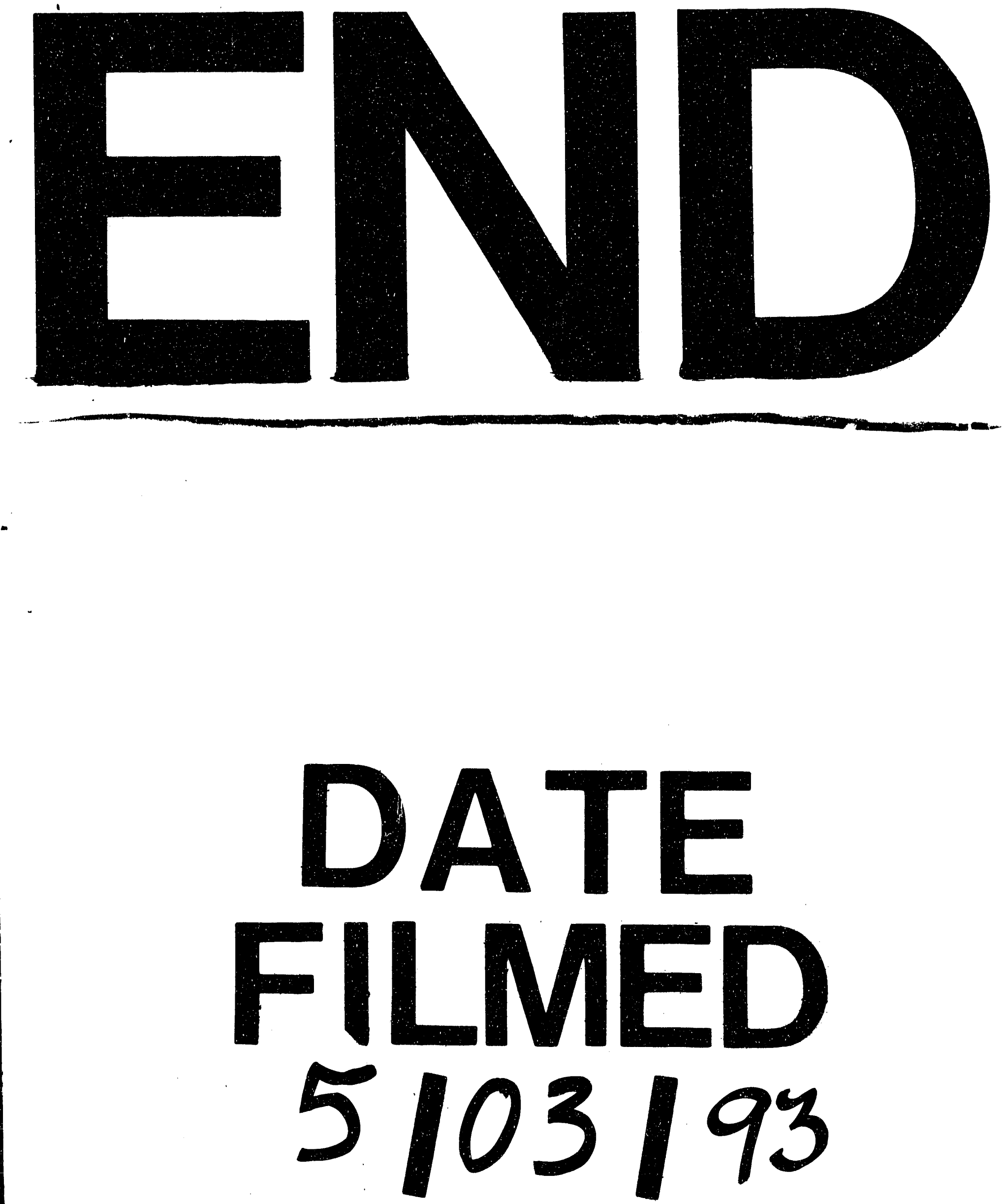
